\title{
Declining Dynamism, Increasing Markups and Missing Growth: The Role of the Labor Force*
}

\author{
Michael Peters $^{\dagger} \quad$ Conor Walsh ${ }^{\ddagger}$ \\ PRELIMINARY - PLEASE DO NOT CIRCULATE
}

\begin{abstract}
A growing body of empirical research highlights substantial changes in the US economy during the last three decades. Business dynamism - namely job reallocation, firm entry and creative destruction - is declining. Market power, as measured by markups and industry concentration, seems to be on the rise. Aggregate productivity growth is sluggish. We show that declines in the rate of growth of the labor force can qualitatively account for all of these features in a standard model of firm-dynamics. Despite its richness we can characterize the link between population growth and dynamism, markups and growth analytically. When we calibrate the model to the universe of U.S. Census data, the labor force channel can explain a large fraction of the aggregate trends.
\end{abstract}

\section{Introduction}

The U.S. economy is facing substantial challenges. First, a large body of work has documented a decline in business dynamism in recent decades. The start-up rate has fallen substantially (Haltiwanger et al. (2015)), job reallocation is down (Pugsley and Şahin (2015)) and creative destruction has declined. Second, new evidence is emerging that concentration ratios within industries, whether measured by sales or employment, have increased markedly during the same time frame (Autor et al. (2017)), as have measures of markups (De Loecker and Eeckhout (2017)). Lastly, save for the I.T.-fueled boom of the late 1990s, productivity growth has been sluggish for many years (Fernald (2015)).

One leading explanation for the decline in the start-up rate is declining labor force growth, as proposed in Karahan et al. (2016) and Hathaway and Litan (2014). At play are general equilibrium

\footnotetext{
*Jordi Bofill, Farouq Ghandour and Tra Nguyen provided outstanding research assistance. We thank Ilse Lindenlaub for very insightful comments.

†Yale University and NBER. m.peters@yale.edu

$\ddagger$ Yale University. conor.walsh@yale.edu
} 
Figure 1: Labor Force Growth and Start-ups

(a) Labor Force Growth

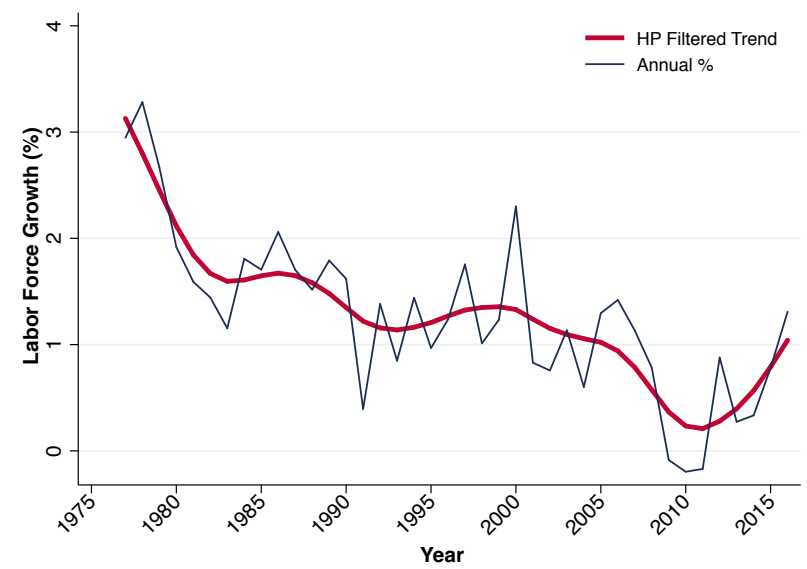

(b) Entry Rate of New Firms

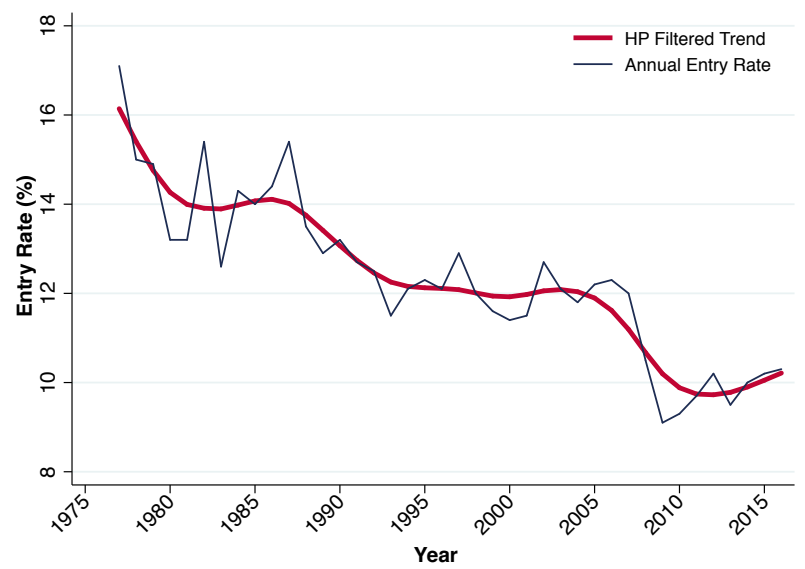

Note: Panel (a) shows the growth in the labor force of the U.S., with the raw series in blue and a HP-filtered trend component in red. The data is sourced from the BLS, accessed through FRED. Panel (b) shows the start-up rate of new firm. The data is from the Business Dynamics Statistics produce by the Center for Economic Studies at the U.S. Census Bureau.

forces. The primary way in which the economy accommodates an influx of new workers is through firm creation. Labor force growth both expands demand and lowers wages, all else equal, raising the incentive to start a new firm. When this slows markedly, as it has since 1970=5 (see Figure 1a), so too will the rate of firm formation. Both the above papers use geographic variation in the age structure of the population of the U.S. to test this idea, and find broad empirical support.

Using the lens of simple growth theory, we show that the implications of declining labor force growth are much broader than the start-up rate alone. The fall in the start-up rate ripples through the economy. Incumbent firms find it easier to raise prices over time, unconstrained by young firms eating at their market share. They also face less competition when they expand into new markets, and grow larger as a result. Average firm size increases, the pace of job reallocation slows, and markups rise across the economy. Lastly, creative destruction declines, and with it the pace of productivity growth falls.

To show this, we present a new model of firm dynamics that is rich enough to match many first-order features of firm growth. In particular, it allows for a fully endogenous determination of markup growth over the life-cycle of the firm, as in Peters (2018). However, it expands the theory beyond a unitary elasticity of substitution, and frees it from counterfactual scale effects, which is vital when dealing with labor force growth. Nonetheless, it remains highly tractable, with tight analytic linkages between labor force growth, creative destruction and markups. We show that declining labor force growth can qualitatively account for all of the above trends.

We then use our theory to quantify the importance of this channel. We calibrate our model to match key moments of the firm lifecycle, focusing on generating the life-cycle patterns of sales and markups which appear in the data. This is of prime importance when considering the dynamic evolution of 
the joint distribution of employment and markups, and sets us apart from other quantitative work exploring the macroeconomic importance of concentration and market power (see e.g. Edmond et al. (2018)). We examine the counterfactual implications of the model under the empirically observed variations in labor force growth, and find they can account for a significant fraction of the observed trends.

Throughout the paper, we take movements in the labor force to be largely exogenous to market concentration and firm dynamics. In the data, slowing labor force growth reflects three primary channels: an end to increasing female participation, declining prime-age male participation, and slowing growth in the working age population (due to both falls in fertility and slowing immigration). In Appendix A.1 we decompose the contributions of each of these channels to the declines observed in Figure $1 \mathrm{a}$, and find that female participation topping out is responsible for the early fall in the rate

of growth, and more recently slow population growth bears much of the blame. While a declining labor share and rising market power may itself have implications for worker participation, here the simplicity of taking these movements as given yields substantial insight into the changing patterns of firm dynamics we see in the data.

\section{Theory}

\subsection{General Environment}

Time is continuous. There is a measure $L_{t}$ of identical households who each supply one unit of labour inelastically. This population of households and hence the labor force is growing at a constant rate $\eta$. Studying the response of markups, growth and business dynamism to changes in $\eta$ is the main focus of this paper. Households have preferences over a final consumption good $c_{t}$, which are given by

$$
U=\int_{0}^{\infty} e^{-\rho t} \ln \left(c_{t}\right) d t
$$

Final consumption is composed of differentiated varieties. As in Klette and Kortum (2004), we model these varieties as particular product lines, which may be produced by multiple firms. Production of the final good takes place in a competitive final sector, with output of the final good given by combining intermediate product lines according to

$$
Y_{t}=\left(\int_{0}^{N_{t}}\left(\sum_{f \in S_{i t}} y_{f i t}\right)^{\frac{\sigma}{\sigma-1}} d i\right)^{\frac{\sigma}{\sigma-1}}
$$

Here $N_{t}$ is the number of active product lines, where these product lines are indexed by $i$. This number evolves endogenously with the creation and destruction of new products. $S_{i t}$ is the set of firms actively producing product $i$ at time $t$, and time $y_{f i t}$ is the production of firm $f$ in product line $i$ at time $t$. Note that this production function implies that the output of firms producing the 
same product are taken as perfect substitutes in the aggregation into final goods, but that different products are imperfect substitutes, with the elasticity of substitution given by $\sigma$.

Firms can be active in multiple product markets. Each firm is characterized by a portfolio of the products they produce, denoted by $\Theta_{f}$, and the productivity with which they produce each product in their portfolio, indexed by $\left\{q_{f i}\right\}_{i \in \Theta_{f}}$. We denote the number of products firm $f$ produces by $n_{f}$. Production of each good uses only labour, and is given by

$$
y_{f i}=q_{f i} l_{f i}
$$

where $l_{f i}$ is the amount of labour hired by firm $f$ to produce product $i$.

Within product markets, firms compete a la Bertrand. By standard arguments, each producer has an optimal markup they would like to charge of $\frac{\sigma}{\sigma-1}$ over marginal cost. However, the presence of competing firms implies that the most efficient producer of the good will resort to limit pricing. If they are unable to price at the optimal markup without inviting competition, they will set their price equal to the marginal cost of the next most efficient producer of that good, who is then indifferent between producing or not. Given the production function in (2), the markup charged in product $i, \mu_{i}$, is given by

$$
\mu_{i}=\min \left\{\frac{\sigma}{\sigma-1}, \frac{q_{i}}{q_{i}^{C}}\right\}
$$

where $q_{i}$ denotes the productivity of current producer in product $i$ and $q_{i}^{C}$ is the productivity of the most efficient competitor.

It is easy verify that product-level sales $p y_{i}$, employment $l_{i}$ and profits $\pi_{i}$ are given by

$$
p y_{i}=\mu_{i}^{1-\sigma}\left(\frac{q_{i}}{w_{t}}\right)^{\sigma-1} Y_{t}, \quad l_{i}=\mu_{i}^{-\sigma}\left(\frac{q_{i}}{w_{t}}\right)^{\sigma-1} \frac{Y}{w^{\prime}}, \quad \pi_{i}=\left(1-\frac{1}{\mu_{i}}\right) \mu_{i}^{1-\sigma}\left(\frac{q_{i}}{w}\right)^{\sigma-1} Y .
$$

For the case of $\mu=\frac{\sigma}{\sigma-1}$ these expressions reduce the usual expressions where profits and labor payments are a constant share of total revenue, i.e. $\pi_{i}=\frac{1}{\sigma} p y_{i}$ and $w l_{i}=\frac{\sigma-1}{\sigma} p y_{i}$. Because firms produce multiple products, firm-level sales are $p y_{f}=\sum_{i=1}^{n_{f}} p y_{i}$ (and for profits and employment analogously).

Given the firm-level allocations in (4), we can also characterize the aggregate allocations. In particular aggregate output $Y_{t}$ and the level of wages $w_{t}$ are given by

$$
Y_{t}=\mathcal{M}_{t} Q_{t} N_{t}^{\frac{1}{\sigma-1}} L_{t}^{P} \text { and } w_{t}=\Lambda_{t} Y_{t} / L_{t}^{P}
$$

where $Q_{t}=\left(\int q_{i}^{\sigma-1} d F_{t}(q)\right)^{\frac{1}{\sigma-1}}=\left(E_{t}\left[q_{i}^{\sigma-1}\right]\right)^{\frac{1}{\sigma-1}}$ and

$$
\mathcal{M}_{t}=\frac{\left(\int_{i} \mu_{i}^{1-\sigma}\left(q_{i} / Q_{t}\right)^{\sigma-1} d i\right)^{\frac{\sigma}{\sigma-1}}}{\int_{i} \mu_{i}^{-\sigma}\left(q_{i} / Q_{t}\right)^{\sigma-1} d i} \quad \text { and } \quad \Lambda_{t}=\frac{\int_{i} \mu_{i}^{-\sigma} d i}{\int_{i} \mu_{i}^{1-\sigma} d i}
$$


The two aggregate statistics $\mathcal{M}_{t}$ and $\Lambda_{t}$ fully summarize the static macroeconomics consequences of monopoly power. Market power reduces both production efficiency (the misallocation term $\mathcal{M}_{t}$ ) and lowers factor prices relative to their social marginal product (the labor wedge $\Lambda_{t}$ ). In particular, a common increase in markups reduces the labor wedge $\Lambda_{t}$ but keeps the allocation efficiency $\mathcal{M}_{t}$ unchanged. The latter is affected by the dispersion of markups. Because our model generates the distribution of markups endogenously, changes in the rate of population growth will directly affect $\mathcal{M}_{t}$ and $\Lambda_{t}$.

\section{Firm Dynamics and Aggregate Growth}

Both firms' productivities and the products they sell evolve endogenously. As in Garcia-Macia et al. (2016), our theory allows for three sources of firm dynamics. These margins will at the same time also determine the rate of aggregate productivity growth and the extent of market power. First we allow for creative destruction by incumbents and entrants (as in Klette and Kortum (2004)). Creative destruction occurs when either an existing firm or a new firm improves the productivity of a product $i$, which is currently produced by another producer. Because the output of firms producing the same product $i$ is considered to be perfectly substitutable (see (1)), such productivity improvements result in churning, whereby the old producer gets replaced.

Second we allow for own-incumbent innovation, whereby firms can improve the productivity $q$ of the products they are currently producing (see Atkeson and Burstein (2010) or Luttmer (2007)). In our model, however, own-incumbent innovation will also be the source of increasing markups as existing firms pull away from their competitors (see (3)).

Third, we allow for the endogenous entry of new varieties. This margin will be source of variety gains, whereby firms can generate product varieties which are entirely new to the economy. As in Young (1998), it is this margin, which implies that the model does not suffer from strong scale effects, i.e. the endogenous growth rate is independent of the size of the population.

We formalize these decisions in the following way. We assume that productivity $q$ evolves on a quality ladder and each successful innovation represents a productivity improvement of $\lambda>1$. We also refer to $\lambda$ as the step size. In order to expand into new products, both incumbent firms and potential entrants make costly investments to choose a Poisson innovation rate. Conditional on successfully creating a new product, this product can either be a new variety or it can improve upon an already existing product from another firm. Importantly, we assume that the direction of innovation is undirected. With probability $\alpha$ the product represents a technological advance over an existing firm, increases the product's productivity by $\lambda$ and forces the current producer to exit. With the complementary productivity $1-\alpha$, the product will be new to the society as a whole. We assume that the quality of new products is given by $q^{\sigma-1}=\omega \bar{q}_{t}$, where $\omega$ is drawn from a fixed distribution $\Gamma(\omega)$ and $\bar{q}_{t}=E_{t}\left[q_{i}^{\sigma-1}\right]$. Hence, as in Buera and Oberfield (2016), the productivity of new varieties is determined both by the existing knowledge embedded in $\bar{q}_{t}$ and by novel ideas. In terms of innovation costs, we assume that incumbent firms pay a $\operatorname{cost} c_{t}^{X}(X, n)$ in units of labour, 
Figure 2: Dynamic Decisions: Entry and Innovation

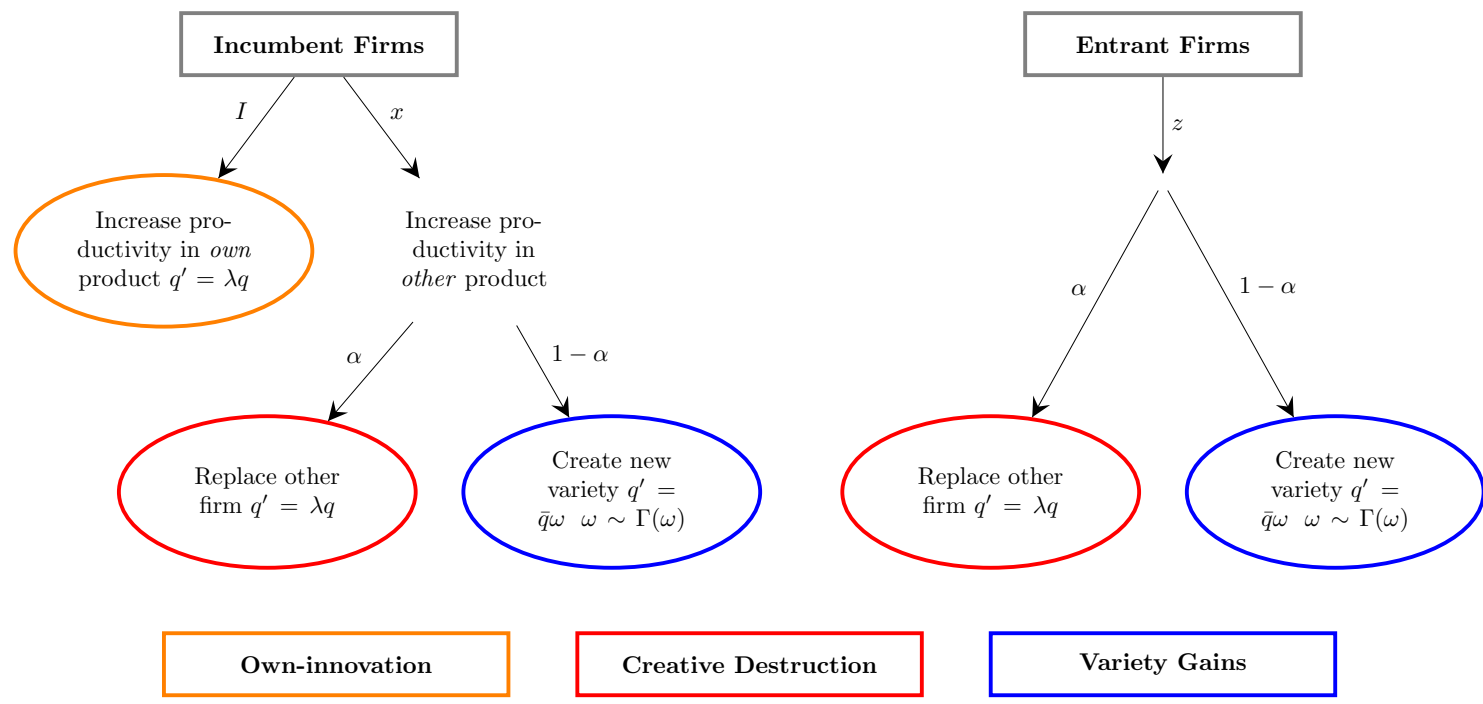

Notes: The figure contains a summary of the dynamic decisions in our model.

where $n$ denotes the number of products the firm is currently producing and $X$ denotes the flow rate of innovation. Entrants have access to a linear entry technology, where each worker generates a flow of $\vartheta_{E}$ ideas.

Firm can also spend resources to engage in own-innovation and improve the productivity of the products in their existing portfolio. For each product, firms can choose a Poisson rate $I_{i}$ to improve the productivity for that product to $\lambda q_{f i}$. They pay a cost $c_{t}^{I}\left(I_{i}, q_{i}\right)$, which is again denominated in units of labour. Figure 2 contains a summary of the innovation and entry decisions in our model.

Finally, we also assume that any product can die exogenously at a constant Poisson rate $\delta$. If a product line dies, the knowledge of how to produce it is lost forever, to all those who knew how to produce it. Allowing for this possibility is not conceptually important but it gives us one additional degree of freedom for our quantitative assesment.

\section{Endogenous Market Power}

A key aspect of our theory is that the distribution of markups is jointly determined with the process of firm-dynamics outlined above. To see why, note that the assumption of the quality ladder structure implies that the equilibrium markup in (3) is given by $\mu_{i}=\min \left\{\frac{\sigma}{\sigma-1}, \lambda^{\Delta_{i}}\right\}$, where $\Delta_{i}$ is the number of quality steps the current producer is ahead of the second most productive firm. We also refer to $\Delta_{i}$ as the productivity gap. As long as $\Delta_{i}<\bar{\Delta} \equiv \frac{\ln (\sigma / \sigma-1)}{\ln \lambda}$, markups are fully determined from limit prices and increasing in $\Delta_{i}$.

To see how the endogenous distribution of markups is determined, note the difference between successful creative destruction and own-innovation. Suppose the current producer of product $i$ has a 
quality gap of $\Delta_{i}$. If this firm is replaced by another producer through successful creative destruction, the quality gaps reduces to unity as the new firm is only a single step ahead of the erstwhile producers. Hence, creative destruction reduces markups. In contrast, if the existing firm successfully increases its productivity through own-innovation, the quality gap increases to $\Delta_{i}+1$ and so do markups. Formally, our model of growth gives rise to an endogenous stochastic process of markups. Let $\tau$ denote the rate of creative destruction and let $I_{i}$ denote the rate of own-innovation. Within a small time interval $\delta_{t}$, the evolution of the markup in product $i$ is given by

$$
\mu_{i t+\delta_{t}}=\left\{\begin{array}{ll}
\lambda & \text { with probabilty } \tau \delta_{t} \\
\lambda \mu_{i t} & \text { with probabilty } I_{i} \delta_{t} \\
\mu_{i t} & \text { with probabilty } 1-\left(\tau+I_{i}\right) \delta_{t}
\end{array} .\right.
$$

Hence, own-innovation is akin to a positive drift for the evolution of markups, while creative destruction is similar to a "reset" shock, which lowers markups. We will show below, that the stochastic process in (6) gives rise to a stationary distribution of markups along the balanced growth path, which explicitly depends on the endogenous rate of creative destruction and own-innovation. And because both these equilibrium outcomes respond to the rate of population growth $\eta$, a declining rate of population growth will directly affect the equilibrium degree of market power. In fact, we will show that lower population growth increases markups.

\section{Equilibrium}

Because firms' innovation decision are forward-looking, we need to characterize the value function. Given the expression for profits in (4) and for markups in (3), the state variables are $\left[\Delta_{i}, q_{i}\right]_{i=1}^{n}$. We denote the rate of creative destruction per product as $\tau_{t}$. This rate is determined endogenously but taken as given by existing firms. Consequently, the value function of a firm is given by

$$
\begin{aligned}
r_{t} V_{t}\left(\left[\Delta_{i}, q_{i}\right]\right)= & \underbrace{\sum_{i=1}^{n} \pi_{t}\left(\left[\Delta_{i}, q_{i}\right]\right)}_{\text {Ptofits }}+\sum_{i=1}^{n} \underbrace{\left(\tau_{t}+\delta_{0}\right)\left[V_{t}\left(\left[\Delta_{j}, q_{j}\right]_{j \neq i}\right)-V\left(\left[\Delta_{i}, q_{i}\right]\right)\right]}_{\text {Creative Destruction and Product Loss }}+\underbrace{\dot{V}_{t}\left(\left[\Delta_{i}, q_{i}\right]\right)}_{\text {Capital Gains }} \\
& +\max _{I} \sum_{i=1}^{n}\left\{I_{i}[\underbrace{V_{t}\left(\left[\Delta_{j}, q_{j}\right]_{j \neq i}\left[\Delta_{i}+1, \lambda q_{i}\right]\right)}_{\text {Value of own innovation }}-V_{t}\left(\left[\Delta_{i}, q_{i}\right]\right)]-c^{I}\left(I_{i}, q_{i}\right) w_{t}\right\} \\
& +\max _{X}\left\{X[\underbrace{\alpha \int V_{t}\left(\left[\Delta_{i}, q_{i}\right], 1, \lambda q\right) d F_{t}}_{\text {Replacing an existing firm }}+\underbrace{(1-\alpha) \int V_{t}\left(\left[\Delta_{i}, q_{i}\right], \bar{\Delta}, \omega \bar{q}_{t}\right)}_{\text {New variety }} d \Gamma-V_{t}\left(\left[\Delta_{i}, q_{i}\right]\right)]-c^{X}(X, n) w_{t}\right\}
\end{aligned}
$$

The free entry condition requires that the expected value of a successful innovation equals the cost of entry

$$
\alpha \int_{q} V_{t}(1, \lambda q) d F_{t}(q)+(1-\alpha) \int_{q} V_{t}\left(\bar{\Delta}, \omega \bar{q}_{t}\right) d \Gamma(\omega)=\frac{1}{\vartheta_{E}} w_{t}
$$


Finally, labor market clearing requires that the total population $L_{t}$ equals the number of production workers $L_{t}^{P}$ and the number of workers engaged in own-innovation, incumbent expansion and entry.

\subsection{The Main Result: Population Growth, Product Creation and Creative Destruction}

In this section we derive the central result of this paper. We show that as long as the economy is on a balanced growth path (BGP), lower population growth reduces the rate of entry and the rate of creative destruction but keeps incumbents' incentives to expand unchanged.

Consider a BGP where the firm size distribution is stationary and all aggregate variables grow at a constant rate. This implies that the number of products $N_{t}$ has to grow at the same rate as the labor force, i.e.

$$
\eta=\frac{\dot{N}_{t}}{N_{t}}
$$

Furthermore, incumbents' expansion rate per product $x$ and the entry flow per product $z$ is constant. This implies that that the rate of creative destruction per product $\tau$ is given by

$$
\tau=\alpha(x+z)
$$

as a share $\alpha$ of entry and expansion activities result in a replacement of existing firms. Similarly, the evolution of the of the number of products $N_{t}$ is given by

$$
\dot{N}_{t}=(1-\alpha)(x+z) N_{t}-\delta N_{t}
$$

These three equations directly imply our main proposition.

Proposition 1. Consider a BGP. The rate of creative destruction $\tau$ is given by

$$
\tau=\frac{\alpha}{1-\alpha}(\eta+\delta)
$$

and the rate of incumbent expansion $x$ and entry $z$ are given by

$$
x=\left(\frac{1}{\zeta} \frac{\vartheta_{x}}{\vartheta_{E}}\right)^{\frac{1}{\zeta-1}} \text { and } z=\frac{\eta+\delta}{1-\alpha}-x
$$

Hence, population growth increases creative destruction, $\frac{\partial \tau}{\partial \eta}>0$, increases the flow rate of entry, $\frac{\partial z}{\partial \eta}>0$, but leaves incumbent expansion unchanged $\frac{\partial x}{\partial \eta}=0$.

Proof. Equation (10) directly follows from (7), (8) and (9). (11) is shown in Section B.2 in the Appendix. 
Proposition 1 contains a key theoretical result of this paper: population growth is absorbed by the economy's extensive margin - entrants do all the work. And because entry is an important component of creative destruction, population growth reduces the extent of creative destruction in equilibrium. Two assumptions, are driving this result. First of all, our assumption that entry and incumbent expansion is undirected, implies that creative destruction is tied to the creation of new products. Secondly, the fact that incumbent expansion $x$ is tied to technology parameters (see (11)) while entry $z$ is determined residually follows from the assumption that incumbents' innovation technology is convex, while entry costs are linear.

Armed with Proposition 1 we now trace out its implications. In particular, we show that lower population growth by reducing creative destruction activities of entrants increases market power, concentration and firm size but lowers the equilibrium entry rate and aggregate productivity growth.

\subsection{Implications: Population Growth, Dynamism, Markups and Growth}

To trace the implications of a reduction in population growth, we start our analysis with a special case of the general framework above by imposing two restrictions. First of all, we assume that consumer preferences take the Cobb Douglas form, i.e. $\sigma=1$. Secondly, we assume the incumbent owninnovation $I$ is exogenous, i.e. existing firms improve the quality of their own products at a constant rate.

This case is analytically attractive because we can solve the entire model in closed form. For our quantitative analysis in Section 3 we are of course not imposing these restrictions. The key simplifications of these assumptions are threefold. First of all, the limit price is always binding, i.e. the markup in product $i$ is given by $\mu_{i}=q_{i} / / q_{i}^{C}=\lambda^{\Delta_{i}}$. Because markups are only constrained by the limit price, we assume that newly invented products charge a markup $\lambda$, i.e. as if they had a productivity advantage of a single step. Secondly, firm sales are not a function of productivity but only of the number of products - see (4). This implies that we can tightly characterize the distribution of sales

and its dependence on $\eta$. Finally, our assumption that $I$ is exogenous allows us to fully characterize both the distribution of markups and the equilibrium growth rate in terms of parameters, including $\eta$.

\section{Result 1: Population Growth and Market Power}

We first turn to the effect of population growth on the equilibrium degree of market power.

Proposition 2. Consider a BGP. Define

$$
\gamma(\eta)=\ln \left(1+\frac{\eta+\delta_{0}}{(1-\alpha) I}\right)
$$

Then: 
1. The stationary distribution of markups is given by

$$
G(\mu)=1-\mu^{-\frac{1}{\ln \lambda} \gamma(\eta)}
$$

2. Aggregate misallocation is given by

$$
\mathcal{M}=e^{-\frac{1}{\gamma(\eta)}} \frac{1+\gamma(\eta)}{\gamma(\eta)} \text { and } \Lambda=\frac{\gamma(\eta)}{1+\gamma(\eta)} .
$$

Hence, lower population growth increases markups and increases misallocation.

Proof. See Section B.4 in the Appendix.

Proposition 2 concisely links the rate of population growth to the stationary degree of market power. Our model implies that the unique equilibrium distribution of markups is pareto distribution, whose shape parameter $\gamma$ is increasing in the rate of population growth. Hence, lower population growth increases both the level and the dispersion of markups.

This result is similar to the finding in Peters (2018), who studies a related model without population growth and without the new variety margin. He shows that the pareto tail of the markup distribution is determined by the rate of creative destruction $\tau$. Proposition 2 leverages this result and the fact that in our economy, creative destruction is directly tied the rate of population growth. The intuition is simple. Recall that creative destruction $\tau$ lowers the expected time a given firm produces a particular product. Hence, as in Jones and Kim (2016), creative destruction plays the role of a "death shock", which limits the extent to which incumbent firms can accumulate market power through successful draws of own-innovation. The resulting stationary distribution of the stochastic process in (6) is the pareto distribution of Proposition 2.

The second part of the proposition contains expressions for the macroeconomic consequences of misallocation. In the case of $\sigma=1$, the two statistics $\mathcal{M}$ and $\Lambda$ only depend on the marginal distribution of markups (see (5)) and can be solved in closed form. It is easy to verify that they are increasing in $\gamma(\eta)$. Lower population growth increases misallocation through lower creative destruction.

\section{Result 2: Population Growth and Aggregate Growth}

As usual in models of Schumpeterian growth, in our economy creative destruction is an important determinant of aggregate productivity growth. This already suggests an important channel why lower population growth might indeed be detrimental for the rate of productivity growth. This intuition is indeed correct.

Proposition 3. Consider a BGP. The growth rate of GDP per capita $g$ is given by

$$
g=\ln (\lambda)(\tau+I)=\ln (\lambda)\left(\frac{\alpha}{1-\alpha}\left(\eta+\delta_{0}\right)+I\right)
$$


Hence, lower population growth reduces productivity growth.

Proof. See Section B.3 in the Appendix.

Proposition 3 shows why population growth is a driver of income growth: it affects creative destruction, which itself is an important component of aggregate productivity growth. Proposition 3 also highlights additional effects, which are absent in this special case of our theory but present in the full model. By assuming that $I$ is exogenous, we abstract from any feedback between creative destruction and incumbent own-innovation. In our full model, I will also be endogenous and hence respond to changes in the rate of population growth. Furthermore, in the Cobb-Douglas case of the model, there are no variety gains, i.e. growth in the number of products does not affect the growth rate of income per capita. Again: variety will be present in our full model. We will show below that the relationship between $g$ and $\eta$ is negative even once these additional aspects are taken into account.

\section{Result 3: Population Growth and Firm Dynamics}

Finally, we turn to the implications for the process of firm-dynamics. We are particularly interested in the the effect of population growth on the start-up rate and average firm size. That lower population growth indeed reduces the start-up rate and increases firm size, follows almost immediately from Proposition 1: lower population is entirely absorbed by the entry of new firms. This naturally reduces the entry rate. At the same time, incumbent firms face less competition from new entrants and hence have an easier time to expand. In a stationary equilibrium this raises average size.

To make these intuitions precise, we need to characterize the distribution of firm size. For the simplified case in this section, it is convenient to measure size by sales. As sales are proportional to the number of products firm sell, we only need to characterize the stationary product distribution. In Section B.5 in the Appendix, we show that this distribution is fully characterized by a simple set of recursive equations. In particular, letting $\omega_{t}(n)$ be the mass of firms with $n$ products, the number of firms is given by $F_{t}=\sum_{n} \omega_{t}(t)$. Stationarity requires that the number of firms grows at the rate of population growth and that therefore $v(n)=\omega_{t}(n) / N_{t}$ is constant. There we also show that $\{v(n)\}_{n}$ is fully characterized by the set of equations

$$
v(n+1)= \begin{cases}\frac{v(1)(\tau+x+\delta+\eta)-z}{2(\tau+\delta)} & \text { if } n=1 \\ \frac{v(n) n(\tau+x+\delta)+v(n) \eta-v(n-1)(n-1) x}{(n+1)(\tau+\delta)} & \text { if } n>1\end{cases}
$$

together with the accounting condition $\sum_{n=1}^{\infty} v(n) n=1$.

Proposition 4. Consider a BGP and let $\{v(n)\}_{n}$ be the distribution of products. Then

$$
\sum_{n=1}^{\infty} v_{n}=\frac{z-v(1)(\tau+\delta)}{\eta}
$$


1. Average firm size is given by

$$
\frac{L_{t}^{P}+L_{t}^{x}}{F_{t}}=\frac{1}{\sum_{n} \nu(n)} \frac{1}{\vartheta_{z}}\left[\frac{\Lambda \lambda}{\lambda-1} \frac{\alpha \eta+\delta+(1-\alpha)\left(\rho+\left(\frac{\lambda-1}{\lambda}\right) I\right)}{(\alpha \eta+\delta+(1-\alpha)(\rho+I))}\left(\frac{(1-\alpha)\left(\rho-\left(\frac{\zeta-1}{\zeta}\right) x\right)+\alpha \eta+\delta}{(1-\alpha)}\right)+\frac{1}{\zeta} x\right],
$$

where $\Lambda$ is given in Proposition 2.

2. The start-up rate is given by

$$
E R=\frac{z N_{t}}{F_{t}}=\frac{z}{\sum_{n} v(n)}
$$

Proof. See Section B.7 in the Appendix.

\section{Quantifying the Effects of Lower Labor Force Growth}

In this Section, we quantify the impact of declining labor force growth on the trends we have outlined. We begin by showing the implications in the simple case discussed above, with Cobb-Douglas aggregation and an exogenous innovation rate $I$ within products. This captures several key patterns well, including the growth in average firm size and the firm entry rate. We then move to the full model, where the endogenous movement in own-incumbent innovation becomes key to generating increases in markups that can speak to the rises we have seen at the economy wide level.

Our exercise is conceptually simple. We calibrate the model to a balanced growth path matching key moments of the data from 1980, when labor force growth was $1.8 \%$. We then vary labor force growth by dropping it $1 \%$, the magnitude of the decline observed until 2008, and trace out the implications for the entry rate, average firm size, markups and lifecycle growth in the sequence of BGP's parameterised by $\eta$.

\subsection{Calibration}

A central element of our work is building a quantitatively accurate picture of the dynamic evolution of sales, employment and markups at the firm-level. Here we show how these rich patterns are generated from the interactions of a few key parameters. The model has an attractive recursive structure which allows identification of these parameters sequentially, by matching central moments of firm dynamics to their analytical expressions in the model.

The parameters we need to calibrate in the simple model appear in Table 1. All but two are determined internally for the simple case. One key parameter we fix is $\alpha$, the share of products that are stolen from other firms when a new product is created. In the full model, this parameter pins down the level of the average markups that young firms charge, since it governs the fraction of firms that immediately charge the monpolistically competitive markup upon creating a product. There is no such analogous information in the simple case, and so we fix it ex-ante at the value we use in the full 
Table 1: Key Parameters

\begin{tabular}{cccc}
\hline \hline Parameter & Description & Baseline Value & Source/Moment \\
\hline \hline$\eta$ & Rate of labor force growth in 1980 & 0.018 & Data from BLS \\
$\delta$ & Destruction rate of products & 0.01 & Entry Rate of $14 \%$ \\
$\lambda$ & Step size on quality ladder & 2 & Markup growth of 10\% between $2.5 \& 12.5$ yrs \\
$I$ & Innovation rate of incumbents & 0.79 & Aggregate growth of $2 \%$ \\
$\vartheta_{X}$ & Cost of product expansion & 0.11 & Avg. sales growth of 50 \% between $2.5 \& 12.5$ yrs \\
$\vartheta_{E}$ & $\quad$ Cost of entry & 0.31 & Avg. firm size of 20.5 from the BDS \\
$\zeta$ & Curvature of innovation cost & 2 & Expansion rates of incumbents (normalisation) \\
$\alpha$ & Fraction of products stolen & 0.5 & Markups of young firms \\
\hline \hline
\end{tabular}

model. The second is the curvature of the innovation cost function, $\zeta$, which we fix at 2 so that cost function is quadratic. This parameter is not identified independent of $\varrho_{x}$, which jointly determine the rates of incumbent expansion. Crucially for our purposes, since incumbent expansion is unaffected by variations in labor force growth (see Proposition, we can set one of these parameters freely.

We begin with determining the rate of creative destruction $\tau$. Together with knowledge of the product entry rate $z$, in the simple model this completely determines the growth rate of incumbent firms. To see this, note that with Cobb-Douglas aggregation, sales of each firm is simply proportional to the number of products each firm has, as in Equation (4). The arrival rate of new products $x$ and their death rate $\delta$ are linear functions of $\tau$ and $z$, and are all that is needed to characterize life-time sales growth (see Equation (??) in Section B.9 in the Appendix). We target an expansion rate of 50\% between ages 2.5. and 12.5, matching evidence from XXX. We also target a firm entry rate of $14 \%$, the rate in 1980 from the BDS, using the expression in (13).

This gives us numbers for $\tau$ and $z$. From equation (8) we get the rate of product death $\delta$, and then from (10) we can solve for the expansion rate per product $x$ by incumbents. With this in hand, we calibrate the innovation step size $\lambda$ to match the observed markup life-cycle growth. As we show in Section B.10 in the Appendix, we can derive a closed form expression for the life-cycle profile of markups. We target a growth in markups between ages 2.5 and 12.5 of $10 \%$, using evidence from XXX. The lifecycle profiles of sales and markups are shown in Figure (3). The average markups across the firm age distribution is 1.1, in line with the cost-weighted average markup calculated by Edmond et al. (2018).

Given this knowledge, all we need to determine the aggregate growth rate $g$ from Equation (12) is $I$, the innovation rate of incumbent firms, which recall is fixed in the simple case. We set a growth rate of $2 \%$ p.a., matching data from the decade of 1975-1985. The last thing to do is calibrate the expansion $\operatorname{cost} \vartheta_{X}$ and the entry cost $\vartheta_{E}$. The former is directly implied by the value for $x$, which has already 
Figure 3: Life-Cycle Growth Profiles

(a) Size By Age

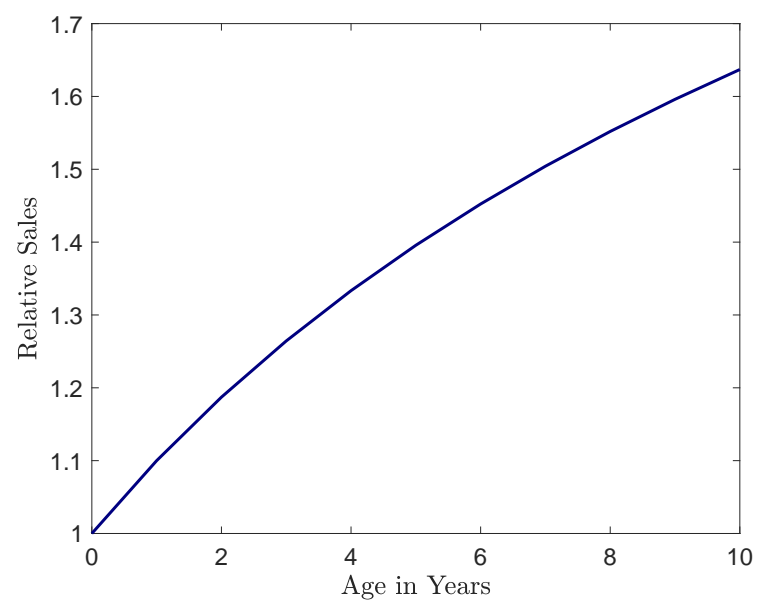

(b) Markups By Age

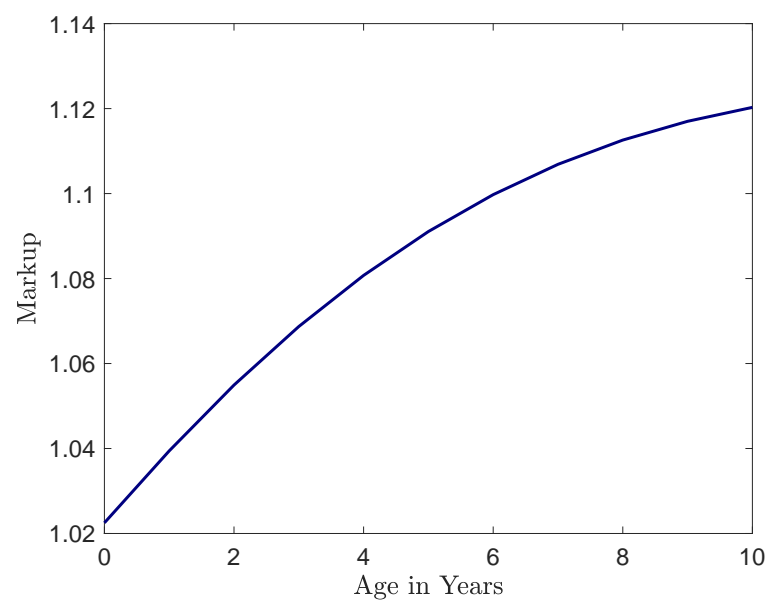

Note: Panel (a) shows the growth in the labor force of the U.S., with the raw series in blue and a HP-filtered trend component in red. The data is sourced from the BLS, accessed through FRED. Panel (b) shows the start-up rate of new firm. The data is from the Business Dynamics Statistics produce by the Center for Economic Studies at the U.S. Census Bureau.

been set. The latter we choose in order to pick average firm size from (??), which we set at 20.5 from the BDS. As we stress throughout, the entry cost has implications only for the number of operational firms in equilibrium, not the entry rate or other lifecycle moments.

\subsection{The Labor Force Slowdown in the Simple Case}

Using the calibrated model, we now examine the implications of a $1 \%$ slow down in labor force growth by comparing BGP's and holding all other parameters constant. In the first panel of Figure 4 we show the effect on average firm size. In the model this rises by almost $11 \%$. To see why, note that incumbents expansion effort is unaffected by population growth. With the rate $x$ unchanging but the rate of creative destruction $\tau$ shrinking, each product an incumbent has survives destruction for longer. As a result, incumbent firms expand in every age bracket. The change in average firm size almost exactly mirrors what is found in the data. In Appendix A.2 we show the time series for average firm size from the BDS, which turns out to have risen $14 \%$ since 1980.

In the top right panel we plot the entry rate. This too experiences a decline which closely matches the data. As discussed above, the economy absorbs all of the decline in labor force growth though a slowing of new entrants per product, $z$. Now since all entrants are new firms, but existing firms may have more than one product, the entry rate of new firms must respond more than the entry rate per product. As such, we see a significant decline in the firm entry rate from a seemingly minor reduction in labor force growth.

However, the simple case misses an important dimension of the data. Average markups at the product level only rise by $1 \%$, much less than the roughly $10 \%$ reported with cost weighting in Edmond 
Figure 4: Slowing Labor Force Growth- Simple Case
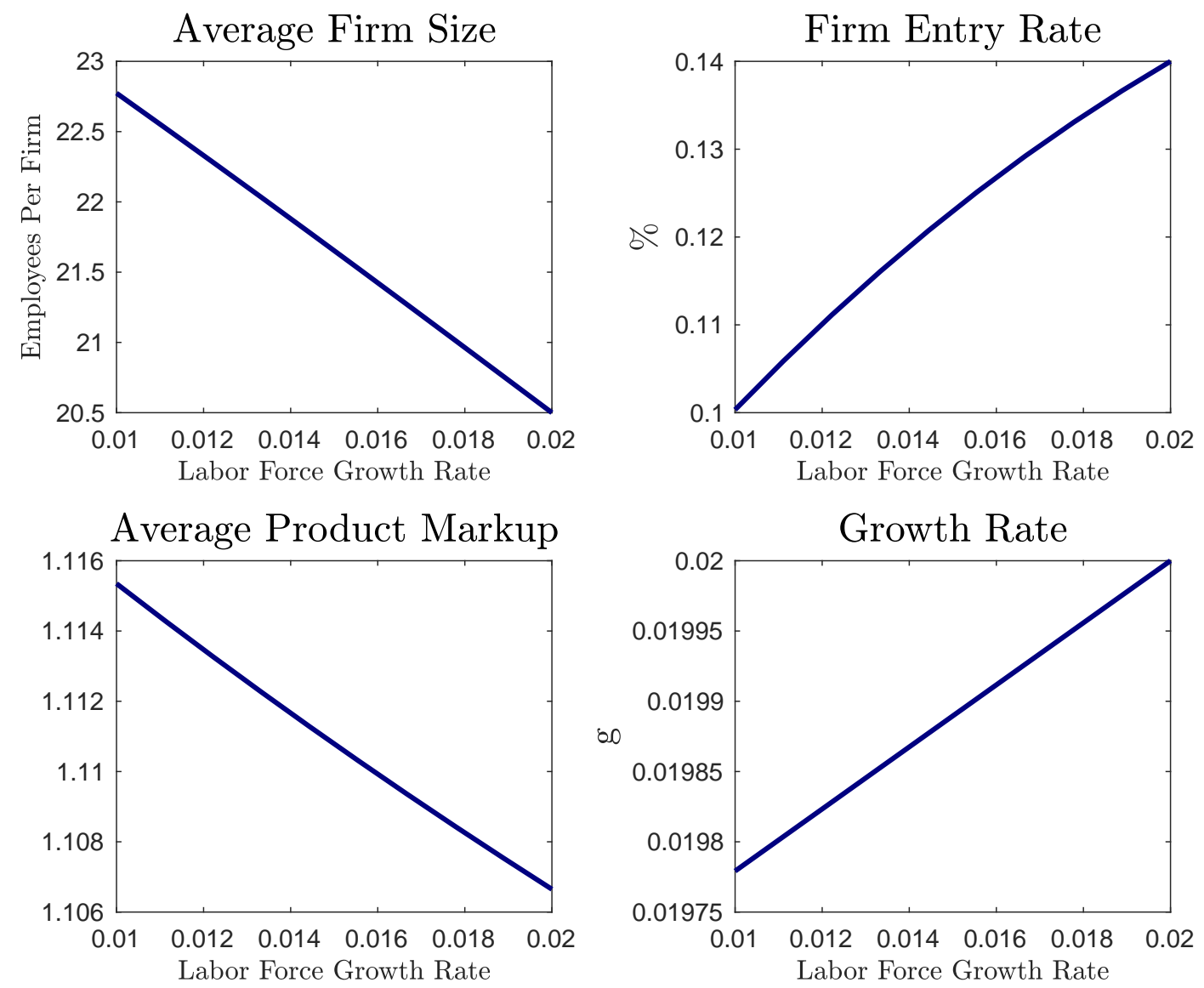

Note: This figure compares central outcomes across BGP's generated by keeping all calibrated parameters constant and varying $\eta$ linearly between 0.01 and 0.02 . 
et al. (2018). Examining equation $X$, declines in labor force growth on the order we are studying having only a minor direct effect on the tail index of the Pareto distribution for markups. Thus a slowdown in creative destruction cannot directly be responsible for the rise in markups we observe.

Indirectly, however, there is an important second effect which appears when we allow I to be chosen optimally. with each product expected to be around for longer before it becomes obsolete, the returns to investing in improving the markup of that product are higher. As such, as $\eta$ slows, firms will increase their incumbent innovation. This serves as a second force depressing the Pareto index for the markup distribution.

\subsection{Moving to the Full Model}

\section{ANALYSIS TO COME}

\section{Conclusion}

We have considered the implications of declining labor force growth in previous decades for a host of troubling macroeconomic outcomes. Operating through a decline in the startup rate, a slowdown in labor force growth causes a rise in markups and concentration. This may provide a new impetus to look a the welfare costs of declining dynamism. Future work might consider optimal policies that take into account the lifecycle nature of firms investment in raising markups, and ask whether attempting to ameliorate these trends should be a goal for policymakers. 


\section{References}

AtKeson, A. And A. Burstein (2010): "Innovation, Firm Dynamics, and International Trade," Journal of Political Economy, 118, 433-484.

Autor, D., D. Dorn, L. F. Katz, C. Patterson, J. VAn Reenen, ET AL. (2017): The fall of the labor share and the rise of superstar firms, National Bureau of Economic Research.

BuerA, F. J. AND E. Oberfield (2016): “The global diffusion of ideas,” Tech. rep., National Bureau of Economic Research.

DE LOECKER, J. AND J. EECKHOUT (2017): “The rise of market power and the macroeconomic implications," Tech. rep., National Bureau of Economic Research.

Edmond, C., V. Midrigan, AND D. Y. XU (2018): “How Costly Are Markups?” Tech. rep., National Bureau of Economic Research.

FERnAlD, J. G. (2015): “Productivity and Potential Output Before, During, and After the Great Recession," NBER Macroeconomics Annual, 29, 1-51.

Garcia-Macia, D., C.-T. Hsieh, AND P. KlenOW (2016): “How Destructive is Innovation?” Working Paper.

Haltiwanger, J., R. Decker, AND R. JARMin (2015): “Top ten signs of declining business dynamism and entrepreneurship in the US," in Kauffman Foundation New Entrepreneurial Growth Conference, Citeseer.

Hathaway, I. AND R. E. LitAn (2014): “What's Driving the Decline in the Firm Formation Rate? A Partial Explanation," The Brookings Institution.

JONES, C. AND J. KIM (2016): “A Schumpeterian Model of Top Income Inequality,” Working Paper.

Karahan, F., B. Pugsley, And A. Sahin (2016): "Demographic Origins of the Startup Deficit," in Technical Report, New York Fed, mimeo.

Klette, T. J. And S. Kortum (2004): “Innovating Firms and Aggregate Innovation," Journal of Political Economy, 112, 986-1018.

LUTTMER, E. G. (2007): "Selection, growth, and the size distribution of firms," The Quarterly Journal of Economics, 122, 1103-1144.

Peters, M. (2018): “Heterogeneous Mark-Ups, Growth and Endogenous Misallocation,” Working Paper.

Pugsley, B. W. AND A. ŞAHIN (2015): “Grown-up business cycles," The Review of Financial Studies.

YOUNG, A. (1998): “Growth without scale effects," Journal of political economy, 106, 41-63. 


\title{
A Empirical Analysis
}

\section{A.1 Labor Force Growth Decomposition}

\author{
ANALYSIS TO COME
}

\section{A.2 Average Firm Size}

Figure 5: Average Firm Size

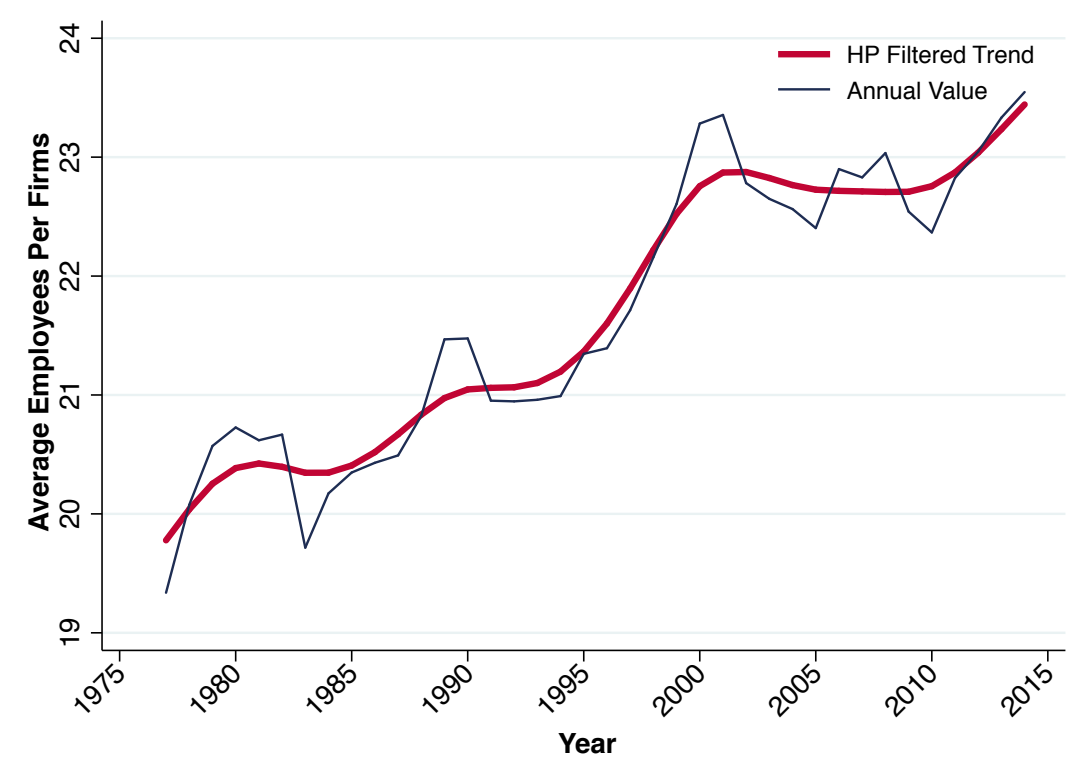

Note: This figure shows average firm size annually, computed as aggregate employment divided by total number of firms, with the raw series in blue and a HP-filtered trend component in red. . The data is from the Business Dynamics Statistics produce by the Center for Economic Studies at the U.S. Census Bureau. 


\section{B Characterizing the special case with $\sigma=1$}

\section{B.1 Static Allocation and the Value function}

Static Allocations This final good, which I take to be the numeraire, is a Cobb-Douglas composite of a continuum of differentiated varieties

$$
\ln Y_{t}=\int_{0}^{N_{t}} \ln \left(\sum_{f \in S_{i t}} y_{f i t}\right) d i
$$

where $N_{t}$ is the number of varieties available at time $t$. This final good is the numeraire. Hence, sales per variety $i$ are given by

$$
p_{i} y_{i}=\frac{Y_{t}}{N_{t}}
$$

The mass of production workers is

$$
L_{P t}=\int_{i=0}^{N_{t}} l_{i t} d i=\frac{Y_{t}}{w_{t}}\left(\frac{1}{N_{t}} \int_{i=0}^{N_{t}} \mu_{i}^{-1} d i\right)
$$

Given that the final good is the numeraire, we have that

$$
w_{t}=\exp \left(\frac{1}{N_{t}} \int_{i=0}^{N_{t}} \ln q_{i t} d i\right) \exp \left(\frac{1}{N_{t}} \int_{i=0}^{N_{t}} \ln \mu_{i t}^{-1} d i\right)
$$

Hence,

$$
Y_{t}=\frac{w_{t}}{\left(\frac{1}{N_{t}} \int_{i=0}^{N_{t}} \mu_{i}^{-1} d i\right)} L_{P t}=\exp \left(\frac{1}{N_{t}} \int_{i=0}^{N_{t}} \ln q_{i t} d i\right) \frac{\exp \left(\frac{1}{N_{t}} \int_{i=0}^{N_{t}} \ln \mu_{i t}^{-1} d i\right)}{\left(\frac{1}{N_{t}} \int_{i=0}^{N_{t}} \mu_{i}^{-1} d i\right)} L_{P t}
$$

Let us denote the distribution of qualities $q$ by $F_{t}(q)$ and the distribution of markups $\mu$ by $G_{t}(\mu)$. Then

$$
Q_{t}=\exp \left(\frac{1}{N_{t}} \int_{i=0}^{N_{t}} \ln q_{i t} d i\right)=\exp \left(\int_{\mathbb{R}_{+}} \ln q d F_{t}(q)\right)
$$

and

$$
\begin{aligned}
\exp \left(\frac{1}{N_{t}} \int_{i=0}^{N_{t}} \ln \mu_{i t}^{-1} d i\right) & =\exp \left(\int \ln \mu^{-1} d G_{t}(\mu)\right)=\exp \left(E\left[\ln \mu_{i t}^{-1}\right]\right) \\
\frac{1}{N_{t}} \int_{i=0}^{N_{t}} \mu_{i}^{-1} d i & =\int \mu^{-1} d G_{t}(\mu)=E\left[\mu_{i t}^{-1}\right]
\end{aligned}
$$

Then

$$
\begin{aligned}
Y_{t} & =Q_{t} \mathcal{M}_{t} L_{P t} \\
w_{t} & =\frac{Y_{t}}{L_{P t}} \Lambda_{t}
\end{aligned}
$$


where

$$
\mathcal{M}_{t}=\frac{\exp \left(E\left[\ln \mu_{i t}^{-1}\right]\right)}{E\left[\mu_{i t}^{-1}\right]} \text { and } \Lambda_{t}=E\left[\mu_{i t}^{-1}\right] \text {. }
$$

Hence, along a BGP the growth rate of $Y_{t}$ is given by

$$
g_{Y}=g_{Q}+\eta
$$

as $L_{P t}$ grows at rate $\eta$ and $\mathcal{M}_{t}$ is constant in a BGP. Wages grow at rate $g_{Q}$. Along a BGP it will also be the case that the number of varieties $N_{t}$ grows at the rate of the laborforce, i.e

$$
g_{N}=\eta
$$

The Value Function In the case of Cobb-Douglas demand, profits are given by

$$
\pi_{i t}=\left(1-\lambda^{-\Delta_{i}}\right) \frac{Y_{t}}{N_{t}}=\pi_{t}\left(\Delta_{i}\right)
$$

Hence, the quality $q$ is not a state variable. Again, it is easy to verify that the value function is additively separable across products. Hence, consider a single product. The value function is given by

$$
r V_{t}(\Delta)-\dot{V}_{t}(\Delta)=\pi_{t}(\Delta)-(\tau+\delta) V_{t}(\Delta)+I\left(V_{t}(\Delta+1)-V_{t}(\Delta)\right)+\max _{x}\left\{x V_{t}(1)-\frac{1}{\vartheta_{x}} x^{\zeta} w_{t}\right\} .
$$

Conjecture that $V_{t}$ takes the form

$$
V_{t}(\Delta)=\kappa w_{t}+\left(\theta-\beta \lambda^{-\Delta}\right) \frac{Y_{t}}{N_{t}}
$$

where $\beta, \theta$ and $\kappa$ are to be determined. As $Y_{t} / N_{t}$ and $w_{t}$ grow at rate $g$ and $r-g=\rho$, we get that

$$
(\rho+\tau+\delta)\left(\kappa w_{t}+\left(\theta-\beta \lambda^{-\Delta}\right) \frac{Y_{t}}{N_{t}}\right)=\pi_{t}(\Delta)+I \beta \lambda^{-\Delta}\left(\frac{\lambda-1}{\lambda}\right) \frac{Y_{t}}{N_{t}}+\max _{x}\left\{x V_{t}(1)-\frac{1}{\vartheta_{x}} x^{\tau} w_{t}\right\}
$$

Hence,

$$
(\rho+\tau+\delta)\left(\kappa w_{t}+\left(\theta-\beta \lambda^{-\Delta}\right) \frac{Y_{t}}{N_{t}}\right)=\left(1-\lambda^{-\Delta_{i}}\right) \frac{Y_{t}}{N_{t}}+I \beta \lambda^{-\Delta}\left(\frac{\lambda-1}{\lambda}\right) \frac{Y_{t}}{N_{t}}+\frac{\zeta-1}{\vartheta_{x}} x^{\zeta} w_{t},
$$

where

$$
x=\left(\frac{\vartheta_{x}}{\zeta} \frac{V_{1}(x)}{w_{t}}\right)^{\frac{1}{\zeta-1}}
$$


Note $x$ is constant along a BGP. Hence,

$$
\begin{aligned}
(\rho+\tau+\delta) \kappa w_{t} & =\frac{\zeta-1}{\vartheta_{x}} x^{\zeta} w_{t} \\
(\rho+\tau+\delta)\left(\theta-\beta \lambda^{-\Delta}\right) \frac{Y_{t}}{N_{t}} & =\left(1-\lambda^{-\Delta_{i}}\right) \frac{Y_{t}}{N_{t}}+I \beta \lambda^{-\Delta}\left(\frac{\lambda-1}{\lambda}\right) \frac{Y_{t}}{N_{t}}
\end{aligned}
$$

This implies that

$$
\begin{aligned}
\kappa & =\frac{\frac{\zeta-1}{\vartheta_{x}} x^{\zeta}}{\rho+\tau+\delta} \\
\theta & =\frac{1}{\rho+\tau+\delta} \\
\beta & =\frac{1}{\rho+\tau+\delta+\left(\frac{\lambda-1}{\lambda}\right) I}
\end{aligned}
$$

Hence,

$$
V_{t}(\Delta)=\frac{\frac{\zeta-1}{\vartheta_{x}} x^{\zeta} w_{t}}{\rho+\tau+\delta}+\left(\frac{1}{\rho+\tau+\delta}-\frac{1}{\rho+\tau+\delta+\left(\frac{\lambda-1}{\lambda}\right) I} \lambda^{-\Delta}\right) \frac{Y_{t}}{N_{t}} .
$$

Note that (5) implies that

$$
\frac{Y_{t}}{N_{t}}=\frac{w_{t} L_{P t}}{N_{t}} \frac{1}{\Lambda}
$$

Hence,

$$
\begin{aligned}
V_{t}(1) & =\frac{\frac{\zeta-1}{\vartheta_{x}} x^{\zeta} w_{t}}{\rho+\tau+\delta}+\left(\frac{\left(\rho+\tau+\delta+\left(\frac{\lambda-1}{\lambda}\right) I\right)-\frac{1}{\lambda}(\rho+\tau+\delta)}{(\rho+\tau+\delta)\left(\rho+\tau+\delta+\left(\frac{\lambda-1}{\lambda}\right) I\right)}\right) \frac{Y_{t}}{N_{t}} \\
& =\frac{1}{\rho+\tau+\delta}\left[\frac{\zeta-1}{\vartheta_{x}} x^{\zeta}+\frac{\frac{\lambda-1}{\lambda}(\rho+\tau+\delta+I)}{\rho+\tau+\delta+\left(\frac{\lambda-1}{\lambda}\right) I} \frac{L_{P t} / N_{t}}{\Lambda}\right] w_{t} .
\end{aligned}
$$

Free entry condition The free entry condition is given by

$$
V_{t}(1)=\frac{1}{\vartheta_{z}} w_{t}
$$

This has two implications.

1. From (16) we know that

$$
x=\left(\frac{\vartheta_{x}}{\zeta} \frac{V_{1}(x)}{w_{t}}\right)^{\frac{1}{\zeta-1}}=\left(\frac{1}{\zeta} \frac{\vartheta_{x}}{\vartheta_{z}}\right)^{\frac{1}{\zeta-1}}
$$

2. Using (17) we get that

$$
\frac{1}{\vartheta_{z}}=\frac{V_{t}(1)}{w_{t}}=\frac{1}{\rho+\tau+\delta}\left[\frac{\zeta-1}{\vartheta_{x}} x^{\zeta}+\frac{\frac{\lambda-1}{\lambda}(\rho+\tau+\delta+I)}{\rho+\tau+\delta+\left(\frac{\lambda-1}{\lambda}\right) I} \frac{L_{P t} / N_{t}}{\Lambda}\right] .
$$


Note that this equation determines $L_{P t} / N_{t}$ in terms of parameters as $\Lambda$ is fully determined in Proposition 2.

\section{B.2 Population Growth and Creative Destruction (Proof of Proposition 1)}

From (7) and (9) in the main text it follows directly that

$$
\eta=\frac{\dot{N}_{t}}{N_{t}}=(1-\alpha)(x+z)-\delta
$$

Hence, (8) implies that

$$
\tau=\alpha(x+z)=\frac{\alpha}{1-\alpha}(\eta+\delta)
$$

This is (10) in Proposition 1.

To derive (11), note that $x$ is given in (18) and that

$$
z=\frac{\tau}{\alpha}-x=\frac{\eta+\delta}{1-\alpha}-x
$$

\section{B.3 Population Growth and Aggregate Growth (Proof of Proposition 3)}

GDP pc is given by (see (15))

$$
y_{t}=\frac{Y_{t}}{L_{t}}=Q_{t} \mathcal{M}_{t} \frac{L_{P t}}{L_{t}}
$$

Along the BGP, $\mathcal{M}_{t}$ and $\frac{L_{P t}}{L_{t}}$ are constant. Hence, the growth rate of GDP pc is equal to the growth rate $Q_{t}$. Standard arguments imply that this growth rate is given by

$$
g_{Q}=\frac{\dot{Q}_{t}}{Q_{t}}=\ln (\lambda)(\tau+I)
$$

\section{B.4 The Distribution of Markups (Proof of Proposition 2)}

The markup in product $i$ is given by

$$
\mu_{i}=\mu\left(\Delta_{i}\right)=\lambda^{\Delta_{i}}
$$

To determine the distribution of markups we hence only need to characterize the share of products with quality gap $\Delta, W_{t}(\Delta)$.

Consider first $W_{t}(1)$. The law of motion for the mass of products with a quality gap of one is given 
by

$$
\begin{aligned}
\frac{d W_{t}(1)}{d t} & =-(\delta+I+\tau) W_{t}(1)+\tau \sum_{\Delta \geq 1} W_{t}(\Delta)+\text { Flow of new Products } \\
& =-(\delta+I+\tau) W_{t}(1)+\tau N_{t}+\text { Flow of new Products, }
\end{aligned}
$$

where $N_{t}$ is the total number of products. Because products are created at rate $\left(z+x^{*}\right)(1-\alpha)$, the total mass of new products is given by

$$
\text { Flow of new Products }=N_{t}\left(z+x^{*}\right)(1-\alpha)=N_{t} \tau\left(\frac{1-\alpha}{\alpha}\right) .
$$

Hence,

$$
\begin{aligned}
\frac{d W_{t}(1)}{d t} & =-(\delta+I+\tau) W_{t}(1)+\tau N_{t}+N_{t} \tau\left(\frac{1-\alpha}{\alpha}\right) \\
& =-(\delta+I+\tau) W_{t}(1)+\frac{\tau N_{t}}{\alpha}
\end{aligned}
$$

Now consider the mass of products with $\Delta \geq 2$. The evolution of this mass satisfies the law of motion:

$$
\frac{d W_{t}(\Delta)}{d t}=I W_{t}(\Delta-1)-(\tau+I+\delta) W_{t}(\Delta)
$$

Along the BGP $W_{t}(\Delta)$ grows at rate $\eta$. Hence, the above equations reduce to

$$
\begin{aligned}
\eta W_{t}(1) & =-(\delta+I+\tau) W_{t}(1)+\frac{\tau N_{t}}{\alpha} \\
\eta W_{t}(\Delta) & =I W_{t}(\Delta-1)-(\tau+I+\delta) W_{t}(\Delta) .
\end{aligned}
$$

Define $v_{t}(\Delta) \equiv W_{t}(\Delta) / N_{t}=v(\Delta)$ as the share of products with quality gap $\Delta$. Then

$$
\begin{aligned}
\eta v(1) & =-(\delta+I+\tau) v(1)+\frac{\tau}{\alpha} \\
\eta v(\Delta) & =I v(\Delta-1)-(\tau+I+\delta) v(\Delta) .
\end{aligned}
$$

Hence,

$$
\begin{aligned}
v(1) & =\frac{1}{\alpha} \frac{\tau}{\delta+I+\tau+\eta} \\
v(\Delta) & =\frac{I}{\delta+I+\tau+\eta} v(\Delta-1) .
\end{aligned}
$$

It is easy to verify that

$$
v(\Delta)=\frac{1}{\alpha} \frac{\tau}{I}\left(\frac{I}{\delta+I+\tau+\eta}\right)^{\Delta}
$$


Note that

$$
\sum_{\Delta=1}^{\infty} v(\Delta)=\frac{1}{\alpha} \frac{\tau}{I} \sum_{\Delta=1}^{\infty}\left(\frac{I}{\delta+I+\tau+\eta}\right)^{\Delta}=1
$$

as required.

From (20) we can derive the distribution of markups. Note first that

$$
\begin{aligned}
P(\Delta \leq d) & =\sum_{\Delta=1}^{d} v(\Delta)=\sum_{\Delta=1}^{d} \frac{1}{\alpha} \frac{\tau}{I}\left(\frac{I}{\delta+I+\tau+\eta}\right)^{\Delta}=1-\left(\frac{I}{I+\frac{\eta+\delta}{1-\alpha}}\right)^{d} \\
& =1-\left(\frac{1}{1+\frac{\eta+\delta}{(1-\alpha) I}}\right)^{d} \\
& =1-e^{-\gamma(\eta) \times d}
\end{aligned}
$$

where

$$
\gamma(\eta)=\ln \left(1+\frac{\eta+\delta}{(1-\alpha) I}\right)
$$

Hence (treating the distribution as continuous), the distribution of log markups is given by

$$
P(\ln \mu \leq m)=P\left(\Delta \leq \frac{m}{\ln \lambda}\right)=1-e^{-\frac{\gamma(\eta)}{\ln \lambda} m},
$$

i.e. is an exponential distribution. The distribution of markups is therefore given by

$$
\begin{aligned}
P(\mu \leq m) & =P(\ln \mu \leq \ln m)=1-e^{-\frac{\gamma(\eta)}{\ln \lambda} \ln m}=1-e^{\ln m^{-\frac{\gamma(\eta)}{\ln \lambda}}} \\
& =1-m^{-\frac{\gamma(\eta)}{\ln \lambda}} .
\end{aligned}
$$

where the pareto tail of the markup distribution is given in (21). The pareto tail is increasing in $\eta$ and decreasing in $I$.

\section{B.5 The Firm-Size Distribution}

We first derive the firm-size distribution, i.e. the distribution of products across firms. Let $\tau+\delta$ denote the rate of losing a product and $x$ the rate of gaining a product and $z$ the flow rate of entry. Let $\omega_{t}(n)$ be the mass of firms with $n$ products at time $t$. Consider $n \geq 2$. Then

$$
\dot{\omega}_{t}(n)=\underbrace{\omega_{t}(n-1)(n-1) x}_{\text {From } n-1 \text { to } \text { products }}+\underbrace{\omega_{t}(n+1)(n+1)(\tau+\delta)}_{\text {From } n+1 \text { ton products }}-\underbrace{\omega_{t}(n) n(\tau+x+\delta)}_{\text {From } n \text { to } n-1 \text { or } n+1 \text { products }} .
$$

For $n=1$ we have

$$
\dot{\omega}_{t}(1)=z_{t}+\omega_{t}(2) 2(\tau+\delta)-\omega_{t}(1)(\tau+x+\delta)
$$


Along the BGP the mass of firms grows at rate $\eta$. Intuitively: the distribution of firms across products is stationary and the number of products $N_{t}$ is increasing at rate $\eta$. Hence, the mass of firms is increasing at rate $\eta$. Hence, along the BGP we have

$$
\dot{\omega}_{t}(n)=\eta \omega_{t}(n)
$$

This implies

$$
\begin{aligned}
& \eta \omega_{t}(n)=\omega_{t}(n-1)(n-1) x+\omega_{t}(n+1)(n+1)(\tau+\delta)-\omega_{t}(n) n(\tau+x+\delta) \\
& \eta \omega_{t}(1)=z_{t}+\omega_{t}(2) 2(\tau+\delta)-\omega_{t}(1)(\tau+x+\delta) .
\end{aligned}
$$

Rewriting yields

$$
\begin{aligned}
& 0=\omega_{t}(n-1)(n-1) x+\omega_{t}(n+1)(n+1)(\tau+\delta)-\omega_{t}(n) n(\tau+x+\delta)-\omega_{t}(n) \eta \\
& 0=z_{t}+\omega_{t}(2) 2(\tau+\delta)-\omega_{t}(1)(\tau+x+\delta)-\omega_{t}(1) \eta
\end{aligned}
$$

Then

$$
\omega_{t}(2)=\frac{\omega_{t}(1)(\tau+x+\delta+\eta)-z_{t}}{2(\tau+\delta)}
$$

and

$$
\omega_{t}(n+1)=\frac{\omega_{t}(n) n(\tau+x+\delta)+\omega_{t}(n) \eta-\omega_{t}(n-1)(n-1) x}{(n+1)(\tau+\delta)}
$$

These are all homogeneous in $N_{t}$, the number of products. Let $v(n)=\frac{\omega_{t}(n)}{N_{t}}$ and $z=\frac{z_{t}}{N_{t}}$. Then

$$
v(2)=\frac{v(1)(\tau+x+\delta+\eta)-z}{2(\tau+\delta)}
$$

and

$$
v(n+1)=\frac{v(n) n(\tau+x+\delta)+v(n) \eta-v(n-1)(n-1) x}{(n+1)(\tau+\delta)} \quad \text { for } n \geq 2
$$

Recall that $z, \tau$ and $x$ are determined in closed form in the equilibrium. Given $v(1)$, these equations fully determine $[v(n)]_{n \geq 2}$. We can then pin down $v(1)$ from the requirement that

$$
\sum_{n=1}^{\infty} v(n) n=\sum_{n=1}^{\infty} \frac{\omega_{t}(n)}{N_{t}} n=\frac{\sum_{n=1}^{\infty} \omega_{t}(n) n}{N_{t}}=1
$$

Hence, equations (24), (25) and (26) fully determine the firm-size distribution $[v(n)]_{n \geq 1}$. 


\section{B.6 The Allocation of Labor}

Solving for the allocation of production workers From the free entry condition in (19) we get that

$$
\frac{1}{\vartheta_{z}}=\frac{1}{\rho+\tau+\delta}\left[\frac{\zeta-1}{\vartheta_{x}} x^{\zeta}+\frac{\frac{\lambda-1}{\lambda}(\rho+\tau+\delta+I)}{\rho+\tau+\delta+\left(\frac{\lambda-1}{\lambda}\right) I} \frac{L_{P t} / N_{t}}{\Lambda}\right] \text {. }
$$

This implies that

$$
\frac{L_{P t}}{N_{t}}=\Lambda \frac{\rho+\tau+\delta+\left(\frac{\lambda-1}{\lambda}\right) I}{\frac{\lambda-1}{\lambda}(\rho+\tau+\delta+I)}\left(\frac{\rho+\tau+\delta}{\vartheta_{z}}-\frac{\zeta-1}{\vartheta_{x}} x^{\zeta}\right)
$$

Using that $\tau=\frac{\alpha}{1-\alpha}(\eta+\delta)$, we get that

$$
\frac{L_{P t}}{N_{t}}=\Lambda \frac{\lambda}{\lambda-1} \frac{\alpha \eta+\delta+(1-\alpha)\left(\rho+\left(\frac{\lambda-1}{\lambda}\right) I\right)}{(\alpha \eta+\delta+(1-\alpha)(\rho+I))}\left(\frac{(1-\alpha) \rho+\alpha \eta+\delta}{(1-\alpha) \vartheta_{z}}-\frac{\zeta-1}{\vartheta_{x}} x^{\zeta}\right)
$$

Also, using that $x=\left(\frac{1}{\zeta} \frac{\vartheta_{x}}{\vartheta_{z}}\right)^{\frac{1}{\zeta-1}}$ (see (18) we get that

$$
\frac{\zeta-1}{\vartheta_{x}} x^{\zeta}=\frac{1}{\vartheta_{z}}(\zeta-1) \frac{\vartheta_{z}}{\vartheta_{x}}\left(\frac{1}{\zeta} \frac{\vartheta_{x}}{\vartheta_{z}}\right)^{\frac{\zeta}{\zeta-1}}=\frac{1}{\vartheta_{z}}(\zeta-1)\left(\frac{1}{\zeta}\right)^{\frac{\zeta}{\zeta-1}}\left(\frac{\vartheta_{x}}{\vartheta_{z}}\right)^{\frac{1}{\zeta-1}}=\frac{1}{\vartheta_{z}}\left(\frac{\zeta-1}{\zeta}\right)\left(\frac{1}{\zeta} \frac{\vartheta_{x}}{\vartheta_{z}}\right)^{\frac{1}{\zeta-1}} .
$$

Hence,

$$
\frac{L_{P t}}{N_{t}}=\Lambda \frac{\lambda}{\lambda-1} \frac{\alpha \eta+\delta+(1-\alpha)\left(\rho+\left(\frac{\lambda-1}{\lambda}\right) I\right)}{(\alpha \eta+\delta+(1-\alpha)(\rho+I))} \frac{1}{\vartheta_{z}}\left(\frac{(1-\alpha) \rho+\alpha \eta+\delta}{(1-\alpha)}-\left(\frac{\zeta-1}{\zeta}\right) x\right)
$$

Note that

$$
\begin{aligned}
\frac{\partial}{\partial \eta}\left[\frac{\alpha \eta+\delta+(1-\alpha)\left(\rho+\left(\frac{\lambda-1}{\lambda}\right) I\right)}{(\alpha \eta+\delta+(1-\alpha)(\rho+I))}\right] & =\frac{\alpha(\alpha \eta+\delta+(1-\alpha)(\rho+I))-\left(\alpha \eta+\delta+(1-\alpha)\left(\rho+\left(\frac{\lambda-1}{\lambda}\right) I\right)\right) \alpha}{(\alpha \eta+\delta+(1-\alpha)(\rho+I))^{2}} \\
& =\frac{\alpha(1-\alpha) \frac{1}{\lambda} I}{(\alpha \eta+\delta+(1-\alpha)(\rho+I))^{2}}>0 .
\end{aligned}
$$

Hence,

$$
\frac{\partial L_{P, t} / N_{t}}{\partial \eta}>0
$$

because $\frac{\partial \Lambda}{\partial \eta}>0$ and $\frac{\partial \frac{(1-\alpha) \rho+\alpha \eta+\delta}{(1-\alpha)}}{\partial \eta}>0$.

Total firm employment Total employment by firms is given by

$$
L_{t}^{\text {Firm }}=L_{t}^{P}+L_{t}^{X}=L_{t}^{P}+N_{t} \frac{1}{\vartheta_{x}} x^{\zeta}
$$


Hence,

$$
\begin{aligned}
\frac{L_{t}^{\text {Firm }}}{N_{t}} & =\Lambda \frac{\lambda}{\lambda-1} \frac{\alpha \eta+\delta+(1-\alpha)\left(\rho+\left(\frac{\lambda-1}{\lambda}\right) I\right)}{(\alpha \eta+\delta+(1-\alpha)(\rho+I))} \frac{1}{\vartheta_{z}}\left(\frac{(1-\alpha) \rho+\alpha \eta+\delta}{(1-\alpha)}-\left(\frac{\zeta-1}{\zeta}\right) x\right)+\frac{1}{\vartheta_{x}} x^{\zeta} \\
& =\frac{1}{\vartheta_{z}}\left[\Lambda \frac{\lambda}{\lambda-1} \frac{\alpha \eta+\delta+(1-\alpha)\left(\rho+\left(\frac{\lambda-1}{\lambda}\right) I\right)}{(\alpha \eta+\delta+(1-\alpha)(\rho+I))}\left(\frac{(1-\alpha) \rho+\alpha \eta+\delta}{(1-\alpha)}-\left(\frac{\zeta-1}{\zeta}\right) x\right)+\frac{\vartheta_{z}}{\vartheta_{x}} x^{\zeta}\right] \\
& =\frac{1}{\vartheta_{z}}\left[\Lambda \frac{\lambda}{\lambda-1} \frac{\alpha \eta+\delta+(1-\alpha)\left(\rho+\left(\frac{\lambda-1}{\lambda}\right) I\right)}{(\alpha \eta+\delta+(1-\alpha)(\rho+I))}\left(\frac{(1-\alpha) \rho+\alpha \eta+\delta}{(1-\alpha)}-\left(\frac{\zeta-1}{\zeta}\right) x\right)+\frac{1}{\zeta} x\right](27)
\end{aligned}
$$

\section{B.7 Population Growth and Dynamism (Proposition 4)}

The number of firms is given by

$$
F_{t}=\sum_{n} \omega_{t}(n)=N_{t} \sum_{n} v(n)
$$

Hence, the entry rate is given by

$$
E R=\frac{z N_{t}}{F_{t}}=\frac{z}{\sum_{n} v(n)}
$$

Average firm size given by

$$
\begin{aligned}
\text { AvSize }=\frac{L_{t}^{\text {Firm }}}{F_{t}} & =\frac{L_{t}^{\text {Firm }}}{N_{t} \sum_{n} v(n)} \\
& =\frac{1}{\sum_{n} v(n)} \frac{1}{\vartheta_{z}}\left[\Lambda \frac{\lambda}{\lambda-1} \frac{\alpha \eta+\delta+(1-\alpha)\left(\rho+\left(\frac{\lambda-1}{\lambda}\right) I\right)}{(\alpha \eta+\delta+(1-\alpha)(\rho+I))}\left(\frac{(1-\alpha) \rho+\alpha \eta+\delta}{(1-\alpha)}-\left(\frac{\zeta-1}{\zeta}\right) x\right)+\frac{1}{\zeta}\right]
\end{aligned}
$$

where the second equality follows from (27). Note that $L_{t}^{\text {Firm }} / N_{t}$ is increasing in $\eta \cdot{ }^{1}$ Note also that (see (31))

$$
\sum_{n} v(n)=\frac{z-v(1)(\tau+\delta)}{\eta} .
$$

We conjecture that $\sum_{n} v(n)$ is increasing in $\eta$.

\footnotetext{
${ }^{1}$ Recall that $L_{t}^{\text {Firm }} / N_{t}=L_{P, t} / N_{t}+\frac{1}{\vartheta_{x}} x^{\zeta}$ and $L_{P, t} / N_{t}$ is increasing in $\eta$ (see Section B.6).
} 


\section{B.8 Solving for $\sum_{n=1}^{\infty} v(n)$}

Note that many objects of interest only depend on $\sum_{n=1}^{\infty} v(n)$. The distribution $[v(n)]_{n \geq 1}$ is fully determined by (24), (25) and (26), which we replicate here for convenience

$$
\begin{aligned}
v(2) & =\frac{v(1)(\tau+x+\delta+\eta)-z}{2(\tau+\delta)} \\
v(n+1) & =\frac{v(n) n(\tau+x+\delta)+v(n) \eta-v(n-1)(n-1) x}{(n+1)(\tau+\delta)} \text { for } n \geq 2 \\
\sum_{n=1}^{\infty} v(n) n & =1 .
\end{aligned}
$$

From (29) we get that

$$
(n+1)(\tau+\delta) v(n+1)=v(n) n(\tau+x+\delta)+v(n) \eta-v(n-1)(n-1) x
$$

Hence, we sum both sides starting at $n=2$, i.e.

$$
\begin{aligned}
\sum_{n=2}^{\infty}(n+1)(\tau+\delta) v(n+1) & =\sum_{n=2}^{\infty} v(n) n(\tau+x+\delta)+\sum_{n=2}^{\infty} v(n) \eta-\sum_{n=2}^{\infty} v(n-1)(n-1) x \\
(\tau+\delta) \sum_{n=2}^{\infty}(n+1) v(n+1) & =(\tau+x+\delta) \sum_{n=2}^{\infty} v(n) n+\eta \sum_{n=2}^{\infty} v(n)-x \sum_{n=2}^{\infty} v(n-1)(n-1) \\
(\tau+\delta) \sum_{n=3}^{\infty} n v(n) & =(\tau+x+\delta) \sum_{n=2}^{\infty} v(n) n+\eta \sum_{n=2}^{\infty} v(n)-x \sum_{n=1}^{\infty} v(n) n . \\
(\tau+\delta) \sum_{n=3}^{\infty} n v(n) & =(\tau+\delta) \sum_{n=2}^{\infty} v(n) n+x \sum_{n=2}^{\infty} v(n) n+\eta \sum_{n=2}^{\infty} v(n)-x \sum_{n=1}^{\infty} v(n) n \\
0 & =(\tau+\delta) v(2) 2+\eta \sum_{n=2}^{\infty} v(n)-x v(1) .
\end{aligned}
$$

Hence,

$$
(\tau+\delta) v(2) 2=(x+\eta) v(1)-\eta \sum_{n=1}^{\infty} v(n)
$$

Using (28), we get that

$$
v(2) 2(\tau+\delta)=v(1)(\tau+x+\delta+\eta)-z .
$$

This implies that

$$
(x+\eta) v(1)-\eta \sum_{n=1}^{\infty} v(n)=v(1)(\tau+x+\delta+\eta)-z
$$

so that

$$
\sum_{n=1}^{\infty} v(n)=\frac{z-v(1)(\tau+\delta)}{\eta}
$$


This is an equation for $\sum_{n=1}^{\infty} v(n)$ as a function of $v(1)$.

To solve for $v(1)$ and hence, $\sum_{n=1}^{\infty} v(n)$, explicitly, we still need to exploit the requirement $\sum v_{n} n=1$ in (30). Note that (31) does not use (30) yet. To do so, define

$$
m_{n} \equiv v_{n} n,
$$

so that (30) requires that $\sum_{n=1}^{\infty} m_{n}=1$. Again we start from (29), which we can write as

$$
m_{n+1}=m_{n} \frac{(\tau+x+\delta)}{\tau+\delta}+v_{n} \frac{\eta}{\tau+\delta}-m_{n-1} \frac{x}{(\tau+\delta)} \quad \text { for } n \geq 2
$$

Rearranging terms yields

$$
m_{n}=m_{n+1} \frac{\tau+\delta}{\tau+x+\delta}+m_{n-1} \frac{x}{\tau+\delta+x}-v_{n} \frac{\eta}{\tau+x+\delta} \quad \text { for } n \geq 2
$$

Letting

$$
\begin{aligned}
\alpha & =\frac{\tau+\delta}{\tau+\delta+x}<1 \\
\gamma & =\frac{x}{\tau+\delta+x}<1 \\
\beta & =\frac{\eta}{\tau+\delta+x}
\end{aligned}
$$

we can write this equation as

$$
m_{n}=m_{n+1} \alpha+m_{n-1} \gamma-v_{n} \beta \text { for } n \geq 2
$$

Expanding this equation backwards yields

$$
m_{n}=\alpha m_{n+1}+\alpha \sum_{j=2}^{n} \gamma^{n-j+1} m_{j}-\alpha \gamma^{n-1} m_{2}-\beta \sum_{j=1}^{n-1} \gamma^{n-j-1} v_{j+1}+\gamma^{n-1} m_{1} \text { for } n \geq 2 .
$$

Now let us sum this formula starting from $n=2$. This yields

$$
\begin{aligned}
\sum_{n=2}^{\infty} m_{n} & =\alpha \sum_{n=2}^{\infty} m_{n+1}+\alpha \sum_{n=2}^{\infty} \sum_{j=2}^{n} \gamma^{n-j+1} m_{j}-\alpha m_{2} \sum_{j=1}^{\infty} \gamma^{j}-\beta \sum_{n=2}^{\infty} \sum_{j=1}^{n-1} \gamma^{n-j-1} v_{j+1}+m_{1} \sum_{n=1}^{\infty} \gamma^{n} \\
& =\alpha \sum_{n=2}^{\infty} m_{n+1}+\alpha \sum_{n=2}^{\infty} \sum_{j=2}^{n} \gamma^{n-j+1} m_{j}-\beta \sum_{n=2}^{\infty} \sum_{j=1}^{n-1} \gamma^{n-j-1} v_{j+1}+m_{1} \frac{\gamma}{1-\gamma}-\alpha m_{2} \frac{\gamma}{1-\gamma}
\end{aligned}
$$


Now note that

$$
\begin{aligned}
\sum_{n=2}^{\infty} \sum_{j=2}^{n} \gamma^{n-j+1} m_{j} & =\gamma m_{2}+\left(m_{2} \gamma^{2}+m_{3} \gamma\right)+\left(m_{2} \gamma^{3}+\gamma^{2} m_{3}+\gamma m_{4}\right)+\ldots \\
& =m_{2} \sum_{j=1}^{\infty} \gamma^{j}+m_{3} \sum_{j=1}^{\infty} \gamma^{j}+m_{4} \sum_{j=1}^{\infty} \gamma^{j}+\ldots \\
& =\frac{\gamma}{1-\gamma} \sum_{n=2}^{\infty} m_{n} .
\end{aligned}
$$

Similarly, we get that

$$
\begin{aligned}
\sum_{n=2}^{\infty} \sum_{j=1}^{n-1} \gamma^{n-j-1} v_{j+1} & =v_{2}+\left(\gamma v_{2}+v_{3}\right)+\left(\gamma^{2} v_{2}+\gamma v_{3}+v_{4}\right)+\ldots \\
& =v_{2} \sum_{j=0}^{\infty} \gamma^{j}+v_{3} \sum_{j=0}^{\infty} \gamma^{j}+\ldots \\
& =\frac{1}{1-\gamma} \sum_{n=2}^{\infty} v_{n}
\end{aligned}
$$

Substituting these results in (33) yields

$$
\sum_{n=2}^{\infty} m_{n}=\alpha \sum_{n=2}^{\infty} m_{n+1}+\frac{\alpha \gamma}{1-\gamma} \sum_{n=2}^{\infty} m_{n}-\beta \frac{1}{1-\gamma} \sum_{n=2}^{\infty} v_{n}+m_{1} \frac{\gamma}{1-\gamma}-\frac{\alpha \gamma}{1-\gamma} m_{2}
$$

Add $m_{1}$ to both sides to get

$$
\sum_{n=1}^{\infty} m_{n}=\alpha \sum_{n=2}^{\infty} m_{n+1}+\frac{\alpha \gamma}{1-\gamma} \sum_{n=2}^{\infty} m_{n}-\beta \frac{1}{1-\gamma} \sum_{n=2}^{\infty} v_{n}+m_{1}\left(\frac{\gamma}{1-\gamma}+1\right)-\frac{\alpha \gamma}{1-\gamma} m_{2}
$$

Add and subtract $\frac{a \gamma}{1-\gamma} m_{1}$ on the right hand side to get

$$
\sum_{n=1}^{\infty} m_{n}=\alpha \sum_{n=2}^{\infty} m_{n+1}+\frac{\alpha \gamma}{1-\gamma} \sum_{n=1}^{\infty} m_{n}-\beta \frac{1}{1-\gamma} \sum_{n=2}^{\infty} v_{n}+m_{1}\left(\frac{\gamma}{1-\gamma}+1-\frac{a \gamma}{1-\gamma}\right)-\frac{\alpha \gamma}{1-\gamma} m_{2}
$$

Add and subtract $\alpha m_{1}+\alpha m_{2}$ to the right hand side, which gives

$$
\sum_{n=1}^{\infty} m_{n}=\alpha \sum_{n=1}^{\infty} m_{n}+\frac{\alpha \gamma}{1-\gamma} \sum_{n=1}^{\infty} m_{n}-\beta \frac{1}{1-\gamma} \sum_{n=2}^{\infty} v_{n}+m_{1}\left(\frac{\gamma}{1-\gamma}+1-\frac{a \gamma}{1-\gamma}-\alpha\right)-\alpha\left(\frac{\gamma}{1-\gamma}+1\right) m_{2}
$$

Now note that $\sum_{n=1}^{\infty} m_{n}=1$ to write this equation as

$$
1=\alpha+\frac{\alpha \gamma}{1-\gamma}-\frac{\beta}{1-\gamma} \sum_{n=2}^{\infty} v_{n}+m_{1}\left(\frac{1}{1-\gamma}-\frac{a \gamma}{1-\gamma}-\alpha\right)-\alpha\left(\frac{1}{1-\gamma}\right) m_{2}
$$


Now note that $v_{1}=m_{1}$. Hence, add and subtract $\frac{\beta}{1-\gamma} v_{1}$ to get

$$
\begin{aligned}
1 & =\alpha+\frac{\alpha \gamma}{1-\gamma}-\frac{\beta}{1-\gamma} \sum_{n=1}^{\infty} v_{n}+m_{1}\left(\frac{1}{1-\gamma}-\frac{a \gamma}{1-\gamma}-\alpha+\frac{\beta}{1-\gamma}\right)-\alpha\left(\frac{1}{1-\gamma}\right) m_{2} \\
& =\alpha+\frac{\alpha \gamma}{1-\gamma}-\frac{\beta}{1-\gamma} \sum_{n=1}^{\infty} v_{n}+m_{1}\left(\frac{1}{1-\gamma}-\frac{\alpha}{1-\gamma}+\frac{\beta}{1-\gamma}\right)-\alpha\left(\frac{1}{1-\gamma}\right) m_{2}
\end{aligned}
$$

Now note that

$$
\alpha=\frac{\tau+\delta}{\tau+\delta+x}=1-\frac{x}{\tau+\delta+x}=1-\gamma
$$

This yields

$$
\begin{aligned}
1 & =\alpha+\gamma-\frac{\beta}{1-\gamma} \sum_{n=1}^{\infty} v_{n}+m_{1}\left(\frac{1}{1-\gamma}-1+\frac{\beta}{1-\gamma}\right)-m_{2} \\
1-\gamma & =\alpha-\frac{\beta}{1-\gamma} \sum_{n=1}^{\infty} v_{n}+m_{1}\left(\frac{\gamma}{1-\gamma}+\frac{\beta}{1-\gamma}\right)-m_{2} \\
0 & =-\frac{\beta}{1-\gamma} \sum_{n=1}^{\infty} v_{n}+m_{1}\left(\frac{\gamma+\beta}{1-\gamma}\right)-m_{2} .
\end{aligned}
$$

Rearranging terms yields

$$
\begin{aligned}
\frac{\beta}{1-\gamma} \sum_{n=1}^{\infty} v_{n} & =m_{1}\left(\frac{\gamma+\beta}{1-\gamma}\right)-m_{2} \\
\sum_{n=1}^{\infty} v_{n} & =m_{1}\left(\frac{\gamma+\beta}{\beta}\right)-\frac{1-\gamma}{\beta} m_{2}
\end{aligned}
$$

From equation (28) we have that

$$
m_{2}=\frac{m_{1}(\tau+x+\delta+\eta)-z}{(\tau+\delta)}
$$

Using the definition of $\gamma$ and $\beta$ we get that

$$
\frac{1-\gamma}{\beta}=\frac{\tau+\delta}{\eta}
$$

Hence,

$$
\frac{1-\gamma}{\beta} m_{2}=\frac{m_{1}(\tau+x+\delta+\eta)-z}{\eta}
$$

This implies that

$$
\sum_{n=1}^{\infty} v_{n}=m_{1}\left(\frac{\gamma+\beta}{\beta}-\frac{\tau+x+\delta+\eta}{\eta}\right)+\frac{1}{\eta} z
$$

Now recall that

$$
\frac{\gamma+\beta}{\beta}=\frac{\frac{x}{\tau+\delta+x}+\frac{\eta}{\tau+\delta+x}}{\frac{\eta}{\tau+\delta+x}}=\frac{x+\eta}{\eta} .
$$


Hence,

$$
\begin{aligned}
\sum_{n=1}^{\infty} v_{n} & =m_{1}\left(\frac{x+\eta}{\eta}-\frac{\tau+x+\delta+\eta}{\eta}\right)+\frac{1}{\eta} z \\
& =-m_{1}\left(\frac{\tau+\delta}{\eta}\right)+\frac{1}{\eta} z .
\end{aligned}
$$

Remembering that $m_{1}=v_{1}$, we have

$$
\sum_{n=1}^{\infty} v_{n}=-v_{1}\left(\frac{\tau+\delta}{\eta}\right)+\frac{1}{\eta} z .
$$

This is the same equation as (31) ....

\section{B.9 The Life-cycle of Sales}

Note that sales is given by $p y_{f t}=\frac{Y_{t}}{N_{t}} n_{f t}$, i.e. sales are proportional to the number of products. Let $p_{n}(a)$ be the prob that a firm has $n$ products at age $a$. This evolves according to

$$
\dot{p}_{n}(a)=(n-1) x p_{n-1}(a)+(n+1)(\tau+\delta) p_{n+1}(a)-n(x+\tau+\delta) p_{n}(a) .
$$

Also, exit is an absorbing state, i.e.

$$
\dot{p}_{0}(a)=(\tau+\delta) p_{1}(a)
$$

This is the same equation as in Klette and Kortum (2004) but where the effective rate of creative destruction is $\tau+\delta$. In particular, the solution is as follows. Let

$$
\gamma(a)=\frac{x\left(1-e^{-(\tau+\delta-x) a}\right)}{\tau+\delta-x \times e^{-(\tau+\delta-x) a}} .
$$

Then

$$
\begin{aligned}
& p_{0}(a)=\frac{\tau+\delta}{x} \gamma(a) \\
& p_{1}(a)=\left(1-p_{0}(a)\right)(1-\gamma(a)) \\
& p_{n}(a)=p_{n-1}(a) \gamma(a)
\end{aligned}
$$

Now note the term $\tau+\delta-x$. We know that $\tau=\alpha(x+z)$. We also note that (along the BGP) $\frac{1-\alpha}{\alpha} \tau=$ $\delta+\eta$. Hence,

$$
\tau+\delta-x=\tau+\frac{1-\alpha}{\alpha} \tau-\eta-x=\frac{1}{\alpha} \tau-\eta-x=z-\eta .
$$

Equation (34) implies a restriction on parameters. To make the firm-size distribution stationary, we need that $\tau+\delta-x<0$. Hence, along the BGP with a stationary solution, we require $z>\eta$. If $\eta=0$ this is always satisfied. With $\eta>0$, the entry flow has to be large enough. Intuitively: if there is too 
little entry, incumbents are "grabbing" all the additional products, which are available because of the larger population size. This makes older firms larger and larger (conditional on survival). Note that this terms also shows in the flow equations for the stationary distribution.

The conditional distribution of $n$ given age $a$ of surviving firms is given by $f_{n}(a) \equiv \frac{p_{n}(a)}{1-p_{0}(a)}$. Hence,

$$
f_{n}(a)=(1-\gamma(a)) \gamma(a)^{n-1}
$$

Hence, the expected log sales of a firm at time $t$ who if of age $a$ are given by

$$
\begin{aligned}
E\left[\ln S_{t}(n) \mid a\right]=\ln \frac{Y_{t}}{N_{t}}+E[\ln n \mid a] & =\ln \frac{Y_{t}}{N_{t}}+\sum_{n=1}^{\infty} \ln n f_{n}(a) \\
& =\ln \frac{Y_{t}}{N_{t}}+\left(\frac{1-\gamma(a)}{\gamma(a)}\right) \sum_{n=1}^{\infty} \ln n \gamma(a)^{n}
\end{aligned}
$$

Note that this expression only depends on $\delta, \tau$ and $x$.

\section{B.10 The Life-cycle of Markups}

Consider the life-cycle of markups. Let $p_{\Delta}(a)$ denote the probability of the product of a given firm having a quality gap $\Delta$ at age $a$. If the firm lost the product, we denote it by a quality gap of 0 . Hence, 0 is an absorbing state.

$$
\dot{p}_{0}(a)=\left(1-p_{0}(a)\right)(\tau+\delta)
$$

Also: firms always enter at 1 . Hence, you can only leave state 1 so that

$$
\dot{p}_{1}(a)=-p_{1}(a)(I+\tau+\delta) .
$$

Finally, for all $\Delta \geq 2$ we have

$$
\dot{p}_{\Delta}(a)=p_{\Delta-1}(a) I-p_{\Delta}(a)(I+\tau+\delta)
$$

The solution to this set of differential equations is given by

$$
\begin{aligned}
p_{0}(a) & =1-e^{-(\tau+\delta) \times a} \\
p_{i+1}(a) & =\left(\frac{1}{i !}\right) I^{i} a^{i}\left(e^{-(I+\tau+\delta) a}\right) \text { for } i \geq 0 .
\end{aligned}
$$

The distribution of quality gaps conditional on survival is then

$$
p_{i+1}^{S}(a) \equiv \frac{p_{i+1}(a)}{1-p_{0}(a)}=\left(\frac{1}{i !}\right) I^{i} a^{i}\left(e^{-I a}\right)
$$

As expected: this does not depend on $\tau$ nor $\delta$. This is a Poisson distribution with parameter $I a$, so that $E[\Delta \mid a]=$ Ia. Now note that $\mu=\lambda^{\Delta}$. Hence, the expected log markup by age is given by

$$
E[\ln \mu \mid a]=\ln \lambda \times E[\Delta \mid a] .
$$


Following the steps in Peters (2018) it can be shows that

$$
E[\Delta \mid a]=1+I \times E\left[a_{P} \mid a_{f}\right]
$$

where

$$
\begin{aligned}
E\left[a_{P} \mid a_{f}\right]= & \frac{\frac{1}{\tau+\delta}\left(1-e^{-(\tau+\delta) a}\right)-\frac{1}{x} e^{-(\tau+\delta) a}\left(1-e^{-x a}\right)}{1-e^{-(x+\tau+\delta) a}}+ \\
& \left(a-\frac{\frac{1}{\tau+\delta}\left(1-e^{-(\tau+\delta) a}\right)-\frac{1}{x} e^{-(\tau+\delta) a}\left(1-e^{-x a}\right)}{1-e^{-(x+\tau+\delta) a}}\right)\left(\frac{(\tau+\delta) e^{-x a}-x e^{-(\tau+\delta) a}}{x\left(1-e^{-(\tau+\delta-x) a}\right)}\right) \ln \left(\frac{\tau+\delta-x \times e^{-(\tau-}}{\tau+\delta-x}\right.
\end{aligned}
$$

Note that $\lim _{a \rightarrow \infty} E\left[a_{P} \mid a\right]=\frac{1}{\tau+\delta}$, which is intuitive because product-firm pairs exit at rate $\tau+\delta$ which makes $\frac{1}{\tau+\delta}$ the expected age. This implies that

$$
\lim _{a \rightarrow \infty} E[\ln \mu \mid a]=\ln \lambda\left(1+\frac{I}{\tau+\delta}\right)=\ln \lambda\left(1+\frac{(1-\alpha) I}{\alpha \eta+\delta}\right),
$$

which shows that the expected markup for very old firms is declining in $\eta$ and increasing in $I$.

\section{B.11 The Life-cycle of Markups}

Consider the life-cycle of markups. Let $p_{\Delta}(a)$ denote the probability of the product of a given firm having a quality gap $\Delta$ at age $a$. If the firm lost the product, we denote it by a quality gap of 0 . Hence, 0 is an absorbing state.

$$
\dot{p}_{0}(a)=\left(1-p_{0}(a)\right)(\tau+\delta)
$$

Also: firms always enter at 1 . Hence, you can only leave state 1 so that

$$
\dot{p}_{1}(a)=-p_{1}(a)(I+\tau+\delta) .
$$

Finally, for all $\Delta \geq 2$ we have

$$
\dot{p}_{\Delta}(a)=p_{\Delta-1}(a) I-p_{\Delta}(a)(I+\tau+\delta)
$$

The solution to this set of differential equations is given by

$$
\begin{aligned}
p_{0}(a) & =1-e^{-(\tau+\delta) \times a} \\
p_{i+1}(a) & =\left(\frac{1}{i !}\right) I^{i} a^{i}\left(e^{-(I+\tau+\delta) a}\right) \text { for } i \geq 0 .
\end{aligned}
$$

The distribution of quality gaps conditional on survival is then

$$
p_{i+1}^{S}(a) \equiv \frac{p_{i+1}(a)}{1-p_{0}(a)}=\left(\frac{1}{i !}\right) I^{i} a^{i}\left(e^{-I a}\right) .
$$


As expected: this does not depend on $\tau$ nor $\delta$. This is a Poisson distribution with parameter $I a$, so that $E[\Delta \mid a]=$ Ia. Now note that $\mu=\lambda^{\Delta}$. Hence, the expected log markup by age is given by

$$
E[\ln \mu \mid a]=\ln \lambda \times E[\Delta \mid a] .
$$

Proposition ?? is shown in Peters (2018). Note that

$$
\lim _{a \rightarrow \infty} E\left[a_{P} \mid a\right]=\frac{1}{\tau+\delta}
$$

This makes sense because products are "dying" at rate $\tau+\delta$ and hence $\frac{1}{\tau+\delta}$ is the expected age. This implies that

$$
\lim _{a \rightarrow \infty} E[\ln \mu \mid a]=\ln \lambda\left(1+\frac{I}{\tau+\delta}\right)
$$

Also

$$
\tau+\delta=\frac{\alpha}{1-\alpha}(\eta+\delta)+\delta=\frac{\alpha}{1-\alpha} \eta+\frac{1}{1-\alpha} \delta
$$

so that

$$
\lim _{a \rightarrow \infty} E[\ln \mu \mid a]=\ln \lambda\left(1+\frac{(1-\alpha) I}{\alpha \eta+\delta}\right) .
$$

Again: the expected markup for old firms is declining in $\eta$.

\section{The General Case}

\section{C.1 The Value function}

The state of the firm is given by $\left[\Delta_{i}, q_{i}\right]_{i=1}^{n}$. Conjecture that the value function is additive, i.e.

$$
V_{t}\left(\left[\Delta_{i}, q_{i}\right]_{i=1}^{n}\right)=\sum_{i=1}^{n} U_{t}\left(\left[\Delta_{i}, q_{i}\right]\right)+n \phi_{t}
$$

which consists of the rents from all the products, plus an expansion value of breaking into new markets. Then

$$
V_{t}\left(\left[\Delta_{j}, q_{j}\right]_{j \neq i}\right)-V_{t}\left(\left[\Delta_{i}, q_{i}\right]_{i=1}^{n}\right)=-U_{t}\left(\left[\Delta_{i}, q_{i}\right]\right)-\phi_{t}
$$

and

$$
V_{t}\left(\left[\Delta_{j}, q_{j}\right]_{j \neq i}\left[\Delta_{i}+1, \lambda q_{i}\right]\right)-V_{t}\left(\left[\Delta_{i}, q_{i}\right]_{i=1}^{n}\right)=U_{t}\left(\left[\Delta+1_{i}, \lambda q_{i}\right]\right)-U_{t}\left(\left[\Delta_{i}, q_{i}\right]\right)
$$

and

$$
\int_{q} V_{t}\left(\left[\Delta_{i}, q_{i}\right]_{i=1}^{n}, 1, \lambda q\right) d F_{t}(q)-V_{t}\left(\left[\Delta_{i}, q_{i}\right]_{i=1}^{n}\right)=\int_{q} U_{t}(1, \lambda q) d F_{t}(q)+\phi_{t}=E_{t}\left[U_{t}(1, \lambda q)\right]+\phi_{t}
$$


Hence we can write this as,

$$
\begin{aligned}
& r \sum_{i=1}^{n} U_{t}\left(\left[\Delta_{i}, q_{i}\right]\right)-\sum_{i=1}^{n}\left(\dot{U}_{t}\left(\left[\Delta_{i}, q_{i}\right]\right)+(r+\tau+\delta) n \phi_{t}-n \dot{\phi}_{t}\right. \\
& =\sum_{i=1}^{n} \pi_{t}\left(\left[\Delta_{i}, q_{i}\right]\right)-\sum_{i=1}^{n}(\tau+\delta) U_{t}\left(\left[\Delta_{i}, q_{i}\right]\right)- \\
& +\sum_{i=1}^{n} I\left[U_{t}\left(\left[\Delta+1_{i}, \lambda q_{i}\right]\right)-U_{t}\left(\left[\Delta_{i}, q_{i}\right]\right)\right] \\
& +\max _{x}\left\{n x\left[\delta_{e} E_{t}\left[U_{t}(1, \lambda q)\right]+\left(1-\delta_{e}\right) \mathbb{E}_{\omega} U_{t}\left(\delta, \omega \bar{q}_{t}\right)+\phi_{t}\right]-n c^{X}\left(\frac{x}{\phi_{e}}, 1\right) w_{t}\right\}
\end{aligned}
$$

where $x=X / n$. Hence, we can write the RHS as

$$
\begin{array}{r}
\sum_{i=1}^{n}\left[\pi_{t}\left(\left[\Delta_{i}, q_{i}\right]\right)-\tau U\left(\left[\Delta_{i}, q_{i}\right]\right)+I\left[U\left(\left[\Delta+1_{i}, \lambda q_{i}\right]\right)-U\left(\left[\Delta_{i}, q_{i}\right]\right)\right]\right] \\
+n \max _{x}\left\{x\left[\delta_{e} \mathbb{E}_{t}\left[U_{t}(1, \lambda q)\right]+\left(1-\delta_{e}\right) \mathbb{E}_{\omega} U_{t}\left(\delta, \omega \bar{q}_{t}\right)+\phi_{t}\right]-c^{X}\left(\frac{x}{\phi_{e}}, 1\right) w_{t}\right\}
\end{array}
$$

Hence, the value function is additive and we can for focus on a single product, which is described by

$$
r U_{t}\left(\left[\Delta_{i}, q_{i}\right]\right)-\dot{U}_{t}\left(\left[\Delta_{i}, q_{i}\right]\right)=\pi_{t}\left(\left[\Delta_{i}, q_{i}\right]\right)-(\tau+\delta) U\left(\left[\Delta_{i}, q_{i}\right]\right)+I\left[U\left(\left[\Delta+1_{i}, \lambda q_{i}\right]\right)-U\left(\left[\Delta_{i}, q_{i}(3 \overline{3}]\right)\right.\right.
$$

and separately solve for the expansion term

$$
(r+\tau+\delta) n \phi_{t}-n \dot{\phi}_{t}=n \max _{x}\left\{x\left[\delta_{e} \mathbb{E}_{t}\left[U_{t}(1, \lambda q)\right]+\left(1-\delta_{e}\right) \mathbb{E}_{\omega} U_{t}\left(\delta, \omega \bar{q}_{t}\right)+\phi_{t}\right]-c^{X}\left(\frac{x}{\phi_{e}}, 1\right) w_{t}\right\}
$$

Solving for $U_{t}\left(\left[\Delta_{i}, q_{i}\right]\right)$ Conjecture that the value function of a particular product takes the following form

$$
U_{t}([\Delta, q])=\frac{u(\Delta)}{g(\sigma-1)+\rho+\tau+\delta} \frac{q^{\sigma-1} Y_{t}}{E\left[\mu^{1-\sigma}\right] N_{t} Q_{t}^{\sigma-1}}
$$

We need to determine the function $u($.$) . Note that Y_{t}$ is growing at rate $g+\eta$ and $Q_{t}^{\sigma-1}$ is growing at rate $g(\sigma-1)$. As such, along the BGP we have

$$
\dot{U}_{t}([\Delta, q])=g(2-\sigma) U_{t}([\Delta, q])
$$

So for a fixed $q$, whether profits are shrinking or rising with growth depends on whether $\sigma \lessgtr 2$. Now, using log preferences we have

$$
r=g+\rho
$$

and combining this with (39) and (37) gives: 


$$
(g(\sigma-1)+\rho+\tau+\delta) U_{t}([\Delta, q])=\pi_{t}([\Delta, q])+I[U([\Delta+1, \lambda q])-U([\Delta, q])]
$$

Using our guess, we have

$$
\begin{aligned}
U_{t}([\Delta+1, \lambda q])-U_{t}([\Delta, q]) & =\left(\frac{u(\Delta+1)}{g(\sigma-1)+\rho+\tau+\delta}(\lambda q)^{\sigma-1}\right) \frac{Y_{t}}{E\left[\mu^{1-\sigma}\right] N_{t} Q_{t}^{\sigma-1}}-\left(\frac{u(\Delta)}{g(\sigma-1)+\rho+\tau+\delta} q^{\sigma-1}\right) \\
& =\frac{u(\Delta+1)(\lambda)^{\sigma-1}-u(\Delta)}{g(\sigma-1)+\rho+\tau+\delta} \frac{q^{\sigma-1} Y_{t}}{E\left[\mu^{1-\sigma}\right] N_{t} Q_{t}^{\sigma-1}}
\end{aligned}
$$

Recall that

$$
Y_{t}=\left[\frac{E\left[\mu_{i}^{1-\sigma}\right]}{E\left[\mu_{i}^{-\sigma}\right]}\right] w_{t} L_{t}^{P}
$$

Define $\Lambda \equiv E\left[\mu_{i}^{-\sigma}\right]^{-1}$. So then

$$
\frac{Y_{t}}{w_{t}}=E\left[\mu_{i}^{1-\sigma}\right] \Lambda \frac{L_{t}^{P}}{N_{t}} N_{t}
$$

or

$$
w_{t}=\frac{Y_{t}}{E\left[\mu_{i}^{1-\sigma}\right] N_{t}}\left(\Lambda \frac{L_{t}^{P}}{N_{t}}\right)^{-1}
$$

where $\frac{L_{t}^{P}}{N_{t}}$ is a key endogenous ratio that we need to solve for on the BGP.

Then

$$
(g(\sigma-1)+\rho+\tau+\delta) U_{t}([\Delta, q])=\pi_{t}([\Delta, q])+I \frac{u(\Delta+1)(\lambda)^{\sigma-1}-u(\Delta)}{g(\sigma-1)+\rho+\tau+\delta} \frac{q^{\sigma-1} Y_{t}}{E\left[\mu^{1-\sigma}\right] N_{t} Q_{t}^{\sigma-1}}
$$

Substituting for $\pi_{t}([\Delta, q])$ and $U_{t}([\Delta, q])$ we get that

$$
(g(\sigma-1)+\rho+\tau+\delta) U_{t}([\Delta, q])=\pi_{t}([\Delta, q])+I \frac{u(\Delta+1)(\lambda)^{\sigma-1}-u(\Delta)}{g(\sigma-1)+\rho+\tau+\delta} \frac{q^{\sigma-1} Y_{t}}{E\left[\mu^{1-\sigma}\right] N_{t} Q_{t}^{\sigma-1}}
$$

The optimality condition for $I$ is given by

$$
\frac{u(\Delta+1)(\lambda)^{\sigma-1}-u(\Delta)}{g(\sigma-1)+\rho+\tau+\delta} \frac{q^{\sigma-1} Y_{t}}{E\left[\mu^{1-\sigma}\right] N_{t} Q_{t}^{\sigma-1}}=\zeta \varrho_{I} \frac{q^{\sigma-1}}{Q_{t}^{\sigma-1}} I^{\zeta-1} w_{t}
$$

This yields

$$
I(\Delta)=\left(\frac{u(\Delta+1)(\lambda)^{\sigma-1}-u(\Delta)}{g(\sigma-1)+\rho+\tau+\delta} \frac{Y_{t}}{E\left[\mu^{1-\sigma}\right] N_{t} w_{t}} \frac{1}{\varrho_{I} \zeta}\right)^{\frac{1}{\zeta-1}}=\left(\frac{u(\Delta+1)(\lambda)^{\sigma-1}-u(\Delta)}{g(\sigma-1)+\rho+\tau+\delta} \frac{1}{\varrho_{I} \zeta} \Lambda \frac{L_{t}^{P}}{N_{t}}\right)^{\frac{1}{\zeta-1}} .
$$


where the second equality uses (40). Hence,

$$
\max _{I}\left\{I \frac{u(\Delta+1)(\lambda)^{\sigma-1}-u(\Delta)}{g(\sigma-1)+\rho+\tau+\delta} \frac{q^{\sigma-1} Y_{t}}{E\left[\mu^{1-\sigma}\right] N_{t} Q_{t}^{\sigma-1}}-\varrho_{I} \frac{q^{\sigma-1}}{Q_{t}^{\sigma-1}} I^{\zeta} w_{t}\right\}=(\zeta-1) \varrho_{I} I(\Delta)^{\zeta} w_{t} \frac{q^{\sigma-1}}{Q_{t}^{\sigma-1}}
$$

Substituting this and our guess into (41) yields

$$
u(\Delta) \frac{q^{\sigma-1} Y_{t}}{E\left[\mu^{1-\sigma}\right] N_{t} Q_{t}^{\sigma-1}}=h(\Delta) q^{\sigma-1} \frac{1}{\mathbb{E}_{t}\left[\mu^{1-\sigma}\right]} \frac{Y_{t}}{Q_{t}^{\sigma-1}} \frac{1}{N_{t}}+(\zeta-1) \varrho_{I} I(\Delta)^{\zeta} w_{t} \frac{q^{\sigma-1}}{Q_{t}^{\sigma-1}},
$$

where $h(\Delta)$ is given in (??). Rearranging terms and using (40) this yields

$$
u(\Delta)=h(\Delta)+(\zeta-1) \varrho_{I} I(\Delta)^{\zeta}\left(\Lambda \frac{L_{t}^{P}}{N_{t}}\right)^{-1}
$$

Substituting the optimal solution for $I$ in (43) yields

$$
u(\Delta)=h(\Delta)+\frac{(\zeta-1) \varrho_{I}}{\left((g(\sigma-1)+\rho+\tau+\delta) \varrho_{I} \zeta\right)^{\frac{\zeta}{\zeta-1}}}\left(u(\Delta+1)(\lambda)^{\sigma-1}-u(\Delta)\right)^{\frac{\zeta}{\zeta-1}}\left(\Lambda \frac{L_{t}^{P}}{N_{t}}\right)^{\frac{1}{\zeta-1}}
$$

This is a difference equation in $u(\Delta)$ given parameters and the general equilibrium statistic $\Lambda \frac{L_{t}^{P}}{N_{t}}$. Given $\{u(\Delta)\}_{\Delta^{\prime}}$ firms' optimal innovation rate is given by

$$
I(\Delta)=\left(\frac{u(\Delta)-h(\Delta)}{(\zeta-1) \varrho_{I}} \Lambda \frac{L_{t}^{P}}{N_{t}}\right)^{1 / \zeta}
$$

\section{C.2 Proof of Proposition 2: The Stationary distribution of markups}

The markup in product $i$ is given by

$$
\mu_{i}=\mu\left(\Delta_{i}\right)=\min \left\{\lambda^{\Delta_{i}}, \frac{\sigma}{\sigma-1}\right\}= \begin{cases}\lambda^{\Delta_{i}} & \text { if } \Delta<\frac{\ln \left(\frac{\sigma}{\sigma-1}\right)}{\ln \lambda} \equiv \bar{\Delta} \\ \frac{\sigma}{\sigma-1} & \text { if } \Delta \geq \frac{\ln \left(\frac{\sigma}{\sigma-1}\right)}{\ln \lambda} \equiv \bar{\Delta}\end{cases}
$$

To determine the distribution of markups we hence only need to characterize the share of products with quality gap $\Delta, W_{t}(\Delta)$.

Consider first $W_{t}(1)$. The law of motion for the mass of products with a quality gap of one is given by

$$
\frac{d W_{t}(1)}{d t}=-(\delta+I+\tau) W_{t}(1)+\tau \sum_{\Delta \geq 1} W_{t}(\Delta)=-(\delta+I+\tau) W_{t}(1)+\tau N_{t}
$$

where $N_{t}$ is the total number of products. Now consider the mass of products with $2 \leq \Delta<\bar{\Delta}$. The 
evolution of this mass satisfies the law of motion:

$$
\frac{d W_{t}(\Delta)}{d t}=I W_{t}(\Delta-1)-(\tau+I+\delta) W_{t}(\Delta)
$$

Finally, consider the states $\Delta \geq \bar{\Delta}$. Because markups are the same in all products with $\Delta \geq \bar{\Delta}$, let $\Omega_{t}=\sum_{\Delta \geq \bar{\Delta}} W_{t}(\Delta)$ be the mass of products with a gap of at least $\bar{\Delta}$. In the interval $[t, t+h]$ this mass evolves according to

$$
\Omega_{t+h}=\Omega_{t}-h(\tau+\delta) \Omega_{t}+h I W_{t}(\bar{\Delta}-1)+\text { Flow of new Products }
$$

as all new products start with the unconstrained markups. Because products are created at rate $\left(z+x^{*}\right)\left(1-\delta_{e}\right)$, the total mass of new products is given by

$$
\text { Flow of new Products }=N_{t} h\left(z+x^{*}\right)\left(1-\delta_{e}\right)=N_{t} \tau h\left(\frac{1-\delta_{e}}{\delta_{e}}\right) .
$$

Hence,

$$
\frac{d \Omega_{t}}{d t}=-(\tau+\delta) \Omega_{t}+I W_{t}(\bar{\Delta}-1)+N_{t} \tau\left(\frac{1-\delta_{e}}{\delta_{e}}\right) .
$$

Along the BGP $W_{t}(\Delta)$ and $\Omega_{t}$ grow at rate $\eta$. Hence, the above three equations reduce

$$
\begin{aligned}
\eta W_{t}(1) & =-(\delta+I+\tau) W_{t}(1)+\tau N_{t} \\
\eta W_{t}(\Delta) & =I W_{t}(\Delta-1)-(\tau+I+\delta) W_{t}(\Delta) \\
\eta \Omega_{t} & =-(\tau+\delta) \Omega_{t}+I W_{t}(\bar{\Delta}-1)+N_{t} \tau\left(\frac{1-\delta_{e}}{\delta_{e}}\right)
\end{aligned}
$$

Define $v_{t}(\Delta) \equiv W_{t}(\Delta) / N_{t}=v(\Delta)$ as the share of products with quality gap $\Delta$ and $\omega \equiv \Omega_{t} / N_{t}$. Then

$$
\begin{aligned}
\eta \nu(1) & =-(\delta+I+\tau) \nu(1)+\tau \\
\eta \nu(\Delta) & =I v(\Delta-1)-(\tau+I+\delta) v(\Delta) \\
\eta \omega & =-(\tau+\delta) \omega+I \nu(\bar{\Delta}-1)+\tau\left(\frac{1-\delta_{e}}{\delta_{e}}\right) .
\end{aligned}
$$

Hence,

$$
\begin{aligned}
v(1) & =\frac{\tau}{\delta+I+\tau+\eta} \\
v(\Delta) & =\frac{I}{\delta+I+\tau+\eta} v(\Delta-1) \\
\omega & =\frac{I}{\delta+\tau+\eta} v(\bar{\Delta}-1)+\frac{\tau}{\delta+\tau+\eta}\left(\frac{1-\delta_{e}}{\delta_{e}}\right)
\end{aligned}
$$


It is easy to verify that

$$
v(\Delta)=\frac{\tau}{I}\left(\frac{I}{\delta+I+\tau+\eta}\right)^{\Delta} .
$$

Now note that

$$
\tau=\frac{\delta_{e}}{1-\delta_{e}}(\eta+\delta) .
$$

Then

$$
\begin{aligned}
v(\Delta) & =\frac{\tau}{I}\left(\frac{I}{\delta+I+\tau+\eta}\right)^{\Delta}=\frac{\frac{\delta_{e}}{1-\delta_{e}}(\eta+\delta)}{I}\left(\frac{I}{\delta+I+\frac{\delta_{e}}{1-\delta_{e}}(\eta+\delta)+\eta}\right)^{\Delta} \\
& =\frac{\delta_{e}}{1-\delta_{e}} \frac{\eta+\delta}{I}\left(\frac{I}{I+\left(\frac{\eta+\delta}{1-\delta_{e}}\right)}\right)^{\Delta} \\
& =\delta_{e} \frac{\frac{\eta+\delta}{1-\delta_{e}}}{I}\left(\frac{I}{I+\left(\frac{\eta+\delta}{1-\delta_{e}}\right)}\right)^{\Delta} \\
& =\delta_{e} \theta\left(\frac{1}{1+\theta}\right)^{\Delta}
\end{aligned}
$$

where

$$
\theta=\frac{\eta+\delta}{\left(1-\delta_{e}\right) I}
$$

Similarly,

$$
\begin{aligned}
\omega & =\frac{I}{\delta+\tau+\eta}\left[\frac{\tau}{I}\left(\frac{I}{\delta+I+\tau+\eta}\right)^{\bar{\Delta}-1}\right]+\frac{\tau}{\delta+\tau+\eta}\left(\frac{1-\delta_{e}}{\delta_{e}}\right) \\
& =\frac{\tau}{\delta+\tau+\eta}\left(\left(\frac{I}{\delta+I+\tau+\eta}\right)^{\bar{\Delta}-1}+\left(\frac{1-\delta_{e}}{\delta_{e}}\right)\right) \\
& =\frac{\frac{\delta_{e}}{1-\delta_{e}}(\eta+\delta)}{\delta+\frac{\delta_{e}}{1-\delta_{e}}(\eta+\delta)+\eta}\left(\left(\frac{I}{\delta+I+\frac{\delta_{e}}{1-\delta_{e}}(\eta+\delta)+\eta}\right)^{\bar{\Delta}-1}+\left(\frac{1-\delta_{e}}{\delta_{e}}\right)\right) \\
& =\frac{\frac{\delta_{e}}{1-\delta_{e}}(\eta+\delta)}{\frac{1}{1-\delta_{e}}(\eta+\delta)}\left(\left(\frac{I}{I+\frac{1}{1-\delta_{e}}(\eta+\delta)}\right)^{\bar{\Delta}-1}+\left(\frac{1-\delta_{e}}{\delta_{e}}\right)\right) \\
& =\delta_{e}\left(\left(\frac{1}{1+\theta}\right)^{\bar{\Delta}-1}+\left(\frac{1-\delta_{e}}{\delta_{e}}\right)\right)^{\bar{\Delta}-1}+\left(1-\delta_{e}\right) \\
& =\delta_{e}\left(\frac{1}{1+\theta}\right)^{\bar{s}}+{ }^{\bar{s}}((\eta)
\end{aligned}
$$


Finally, note that

$$
\begin{aligned}
\sum_{\Delta<\bar{\Delta}} v(\Delta)+\omega & =\sum_{\Delta<\bar{\Delta}} \delta_{e} \theta\left(\frac{1}{1+\theta}\right)^{\Delta}+\delta_{e}\left(\frac{1}{1+\theta}\right)^{\bar{\Delta}-1}+\left(1-\delta_{e}\right) \\
& =\delta_{e} \theta \sum_{\Delta<\bar{\Delta}}\left(\frac{1}{1+\theta}\right)^{\Delta}+\delta_{e}\left(\frac{1}{1+\theta}\right)^{\bar{\Delta}-1}+\left(1-\delta_{e}\right)
\end{aligned}
$$

Now

$$
\sum_{\Delta<\bar{\Delta}}\left(\frac{1}{1+\theta}\right)^{\Delta}=\sum_{\Delta=1}^{\bar{\Delta}-1}\left(\frac{1}{1+\theta}\right)^{\Delta}=\left(\frac{1}{1+\theta}\right) \frac{1-\left(\frac{1}{1+\theta}\right)^{\bar{\Delta}-1}}{1-\left(\frac{1}{1+\theta}\right)}=\frac{1}{\theta}\left(1-\left(\frac{1}{1+\theta}\right)^{\bar{\Delta}-1}\right)
$$

Hence, $\sum_{\Delta<\bar{\Delta}} v(\Delta)+\omega=1$ as required. 


\section{Solving for $\sum_{n=1}^{\infty} v(n)$}

Note that many objects of interest only depend on $\sum_{n=1}^{\infty} v(n)$.

Consider $n \geq 2$. For this branch we have

$$
(n+1)(\tau+\delta) v(n+1)=v(n) n(\tau+x+\delta)+v(n) \eta-v(n-1)(n-1) x
$$

Hence, we sum both sides starting at $n=2$, i.e.

$$
\begin{aligned}
\sum_{n=2}^{\infty}(n+1)(\tau+\delta) v(n+1) & =\sum_{n=2}^{\infty} v(n) n(\tau+x+\delta)+\sum_{n=2}^{\infty} v(n) \eta-\sum_{n=2}^{\infty} v(n-1)(n-1) x \\
(\tau+\delta) \sum_{n=2}^{\infty}(n+1) v(n+1) & =(\tau+x+\delta) \sum_{n=2}^{\infty} v(n) n+\eta \sum_{n=2}^{\infty} v(n)-x \sum_{n=2}^{\infty} v(n-1)(n-1) \\
(\tau+\delta) \sum_{n=3}^{\infty} n v(n) & =(\tau+x+\delta) \sum_{n=2}^{\infty} v(n) n+\eta \sum_{n=2}^{\infty} v(n)-x \sum_{n=1}^{\infty} v(n) n . \\
(\tau+\delta) \sum_{n=3}^{\infty} n v(n) & =(\tau+\delta) \sum_{n=2}^{\infty} v(n) n+x \sum_{n=2}^{\infty} v(n) n+\eta \sum_{n=2}^{\infty} v(n)-x \sum_{n=1}^{\infty} v(n) n \\
0 & =(\tau+\delta) v(2) 2+\eta \sum_{n=2}^{\infty} v(n)-x v(1) .
\end{aligned}
$$

Hence,

$$
(\tau+\delta) v(2) 2=x v(1)-\eta \sum_{n=2}^{\infty} v(n) .
$$

Note that we also have

$$
v(2)=\frac{v(1)(\tau+x+\delta+\eta)-\tilde{z}}{2(\tau+\delta)}
$$

Hence,

$$
v(2) 2(\tau+\delta)=v(1)(\tau+x+\delta+\eta)-\tilde{z}
$$

This implies that

$$
\begin{aligned}
x v(1)-\eta \sum_{n=2}^{\infty} v(n) & =v(1)(\tau+x+\delta+\eta)-\tilde{z} \\
-\eta \sum_{n=1}^{\infty} v(n) & =v(1)(\tau+\delta)-\tilde{z} .
\end{aligned}
$$

so that

$$
\sum_{n=1}^{\infty} v(n)=\frac{z-v(1)(\tau+\delta)}{\eta}
$$


This is an equation for $\sum_{n=1}^{\infty} v(n)$ as a function of $v(1)$. Note that this implies that

$$
\begin{aligned}
\sum_{n=1}^{\infty} v(n)=\frac{z-v(1)(\tau+\delta)}{\eta} & =\frac{\frac{\eta+\delta}{1-\alpha}-x-v(1)\left(\frac{\alpha}{1-\alpha}(\eta+\delta)+\delta\right)}{\eta} \\
& =\frac{\frac{\eta}{1-\alpha}(1-\alpha v(1))+\frac{\delta}{1-\alpha}(1-v(1))-x}{\eta} \\
& =\frac{1}{1-\alpha}(1-\alpha v(1))+\frac{1}{\eta}\left(\frac{\delta}{1-\alpha}(1-v(1))-x\right)
\end{aligned}
$$

If $v(1)$ is increasing in $\eta$ (as it should), this implies that

$$
\begin{gathered}
\frac{\partial \sum_{n=1}^{\infty} v(n)}{\partial \eta}<0 . \\
\tau=\frac{\alpha}{1-\alpha}(\eta+\delta) \\
x=\left(\frac{1}{\zeta} \frac{\vartheta_{x}}{\vartheta_{E}}\right)^{\frac{1}{\zeta-1}} \\
z=\frac{\eta+\delta}{1-\alpha}-x .
\end{gathered}
$$

Define $m_{n}=v_{n} n$ as above.

\section{Start from}

$$
m_{n}=\alpha m_{n+1}+\gamma m_{n-1}-v_{n} \beta
$$

where

$$
\begin{gathered}
\alpha=\frac{\tau+\delta}{\tau+\delta+x}<1 \\
\gamma=\frac{x}{\tau+\delta+x}<1 \\
\beta=\frac{\eta}{\tau+\delta+x}
\end{gathered}
$$

Note this is not what we have above.

Let's expand backwards instead of forwards, to get

$$
\begin{gathered}
m_{n}=\alpha m_{n+1}+\gamma\left(\alpha m_{n}+\gamma m_{n-2}-\beta v_{n-1}\right)-v_{n} \beta \\
m_{n}=\alpha m_{n+1}+\gamma\left(\alpha m_{n}+\gamma\left(\alpha m_{n-1}+\gamma m_{n-3}-\beta v_{n-2}\right)-\beta v_{n-1}\right)-v_{n} \beta
\end{gathered}
$$


We can expand backwards until $n=2$, so we get

$$
m_{n}=\alpha m_{n+1}+\alpha \sum_{j=2}^{n} \gamma^{n-j+1} m_{j}-\alpha \gamma^{n-1} m_{2}-\beta \sum_{j=1}^{n-1} \gamma^{n-j-1} v_{j+1}+\gamma^{n-1} m_{1} .
$$

If we sum the above formula from $n=2$, we get

$$
\begin{aligned}
\sum_{n=2}^{\infty} m_{n} & =\alpha \sum_{n=2}^{\infty} m_{n+1}+\alpha \sum_{n=2}^{\infty} \sum_{j=2}^{n} \gamma^{n-j+1} m_{j}-\alpha m_{2} \sum_{j=1}^{\infty} \gamma^{j}-\beta \sum_{n=2}^{\infty} \sum_{j=1}^{n-1} \gamma^{n-j-1} v_{j+1}+\sum_{n=2}^{\infty} \gamma^{n-1} m_{1} \\
& =\alpha \sum_{n=2}^{\infty} m_{n+1}+\alpha \sum_{n=2}^{\infty} \sum_{j=2}^{n} \gamma^{n-j+1} m_{j}-\beta \sum_{n=2}^{\infty} \sum_{j=1}^{n-1} \gamma^{n-j-1} v_{j+1}+m_{1} \frac{\gamma}{1-\gamma}-\alpha m_{2} \frac{\gamma}{1-\gamma}
\end{aligned}
$$

Now

$$
\begin{aligned}
\sum_{n=2}^{\infty} \sum_{j=2}^{n} \gamma^{n-j+1} m_{j} & =\gamma m_{2}+\left(m_{2} \gamma^{2}+m_{3} \gamma\right)+\left(m_{2} \gamma^{3}+\gamma^{2} m_{3}+\gamma m_{4}\right)+\ldots \\
& =m_{2} \sum_{j=1}^{\infty} \gamma^{j}+m_{3} \sum_{j=1}^{\infty} \gamma^{j}+m_{4} \sum_{j=1}^{\infty} \gamma^{j}+\ldots \\
& =\frac{\gamma}{1-\gamma} \sum_{n=2}^{\infty} m_{n} .
\end{aligned}
$$

and similarly

$$
\begin{aligned}
\sum_{n=2}^{\infty} \sum_{j=1}^{n-1} \gamma^{n-j-1} v_{j+1} & =v_{2}+\left(\gamma v_{2}+v_{3}\right)+\left(\gamma^{2} v_{2}+\gamma v_{3}+v_{4}\right)+\ldots \\
& =v_{2} \sum_{j=0}^{\infty} \gamma^{j}+v_{3} \sum_{j=0}^{\infty} \gamma^{j}+\ldots \\
& =\frac{1}{1-\gamma} \sum_{n=2}^{\infty} v_{n}
\end{aligned}
$$

Now substitute this in (51) to get

$$
\sum_{n=2}^{\infty} m_{n}=\alpha \sum_{n=2}^{\infty} m_{n+1}+\alpha \frac{\gamma}{1-\gamma} \sum_{n=2}^{\infty} m_{n}-\beta \frac{1}{1-\gamma} \sum_{n=2}^{\infty} v_{n}+m_{1} \frac{\gamma}{1-\gamma}-\frac{\alpha \gamma}{1-\gamma} m_{2}
$$

Add $m_{1}$ to both sides to get

$$
\sum_{n=1}^{\infty} m_{n}=\alpha \sum_{n=2}^{\infty} m_{n+1}+\alpha \frac{1}{1-\gamma} \sum_{n=2}^{\infty} m_{n}-\beta \frac{1}{1-\gamma} \sum_{n=2}^{\infty} v_{n}+m_{1}\left(\frac{\gamma}{1-\gamma}+1\right)-\frac{\alpha \gamma}{1-\gamma} m_{2}
$$


Add and subtract $\frac{a}{1-\gamma} m_{1}$ on the right hand side to get

$$
\sum_{n=1}^{\infty} m_{n}=\alpha \sum_{n=2}^{\infty} m_{n+1}+\frac{\alpha}{1-\gamma} \sum_{n=1}^{\infty} m_{n}-\beta \frac{1}{1-\gamma} \sum_{n=2}^{\infty} v_{n}+m_{1}\left(\frac{\gamma-\alpha}{1-\gamma}+1\right)-\frac{\alpha \gamma}{1-\gamma} m_{2}
$$

Another step is to add and subtract $\alpha m_{1}+\alpha m_{2}$ to the right hand side, which gives

$$
\sum_{n=1}^{\infty} m_{n}=\alpha \sum_{n=1}^{\infty} m_{n}+\frac{\alpha}{1-\gamma} \sum_{n=1}^{\infty} m_{n}-\beta \frac{1}{1-\gamma} \sum_{n=2}^{\infty} v_{n}+m_{1}\left(\frac{\gamma-\alpha}{1-\gamma}+1-\alpha\right)-\left(\frac{\alpha \gamma}{1-\gamma}+\alpha\right) m_{2}
$$

Now impose that $\sum_{n=1}^{\infty} m_{n}=1$ to get

$$
\begin{aligned}
1 & =\alpha+\frac{\alpha}{1-\gamma}-\beta \frac{1}{1-\gamma} \sum_{n=2}^{\infty} v_{n}+m_{1}\left(\frac{\gamma-\alpha}{1-\gamma}+1-\alpha\right)-\alpha\left(\frac{\gamma}{1-\gamma}+1\right) m_{2} \\
& =\alpha+\frac{\alpha}{1-\gamma}-\beta \frac{1}{1-\gamma} \sum_{n=2}^{\infty} v_{n}+m_{1}\left(\frac{\gamma-\alpha}{1-\gamma}+1-\alpha\right)-\alpha \frac{1}{1-\gamma} m_{2} .
\end{aligned}
$$

Now add and subtract $\beta \frac{1}{1-\gamma} v_{1}$ on the right hand side

$$
1=\alpha+\frac{\alpha}{1-\gamma}-\beta \frac{1}{1-\gamma} \sum_{n=1}^{\infty} v_{n}+m_{1}\left(\frac{\gamma-\alpha}{1-\gamma}+1-\alpha\right)-\alpha \frac{1}{1-\gamma} m_{2}+\beta \frac{1}{1-\gamma} v_{1}
$$

Now remember that

$$
m_{1}=v_{1} 1=v_{1}
$$

Then

$$
1=\alpha+\frac{\alpha}{1-\gamma}-\beta \frac{1}{1-\gamma} \sum_{n=1}^{\infty} v_{n}+v_{1}\left(\frac{\gamma-\alpha}{1-\gamma}+1-\alpha+\frac{\beta}{1-\gamma}\right)-\alpha \frac{1}{1-\gamma} m_{2}
$$

Now note that

$$
\alpha=\frac{\tau+\delta}{\tau+\delta+x}=1-\frac{x}{\tau+\delta+x}=1-\gamma .
$$

Substituting this above yields

$$
\begin{aligned}
1 & =\alpha+1-\beta \frac{1}{1-\gamma} \sum_{n=1}^{\infty} v_{n}+v_{1}\left(\frac{\gamma}{1-\gamma}-\frac{\alpha}{1-\gamma}+1-\alpha+\frac{\beta}{1-\gamma}\right)-m_{2} \\
0 & =\alpha-\beta \frac{1}{1-\gamma} \sum_{n=1}^{\infty} v_{n}+v_{1}\left(\frac{\gamma}{1-\gamma}-\alpha+\frac{\beta}{1-\gamma}\right)-m_{2} .
\end{aligned}
$$

Note that from (46) we have that

$$
\begin{aligned}
v_{2} & =\frac{v_{1}(\tau+x+\delta+\eta)-\tilde{z}}{2(\tau+\delta)} \\
2 v_{2}(\tau+\delta) & =v_{1}(\tau+x+\delta+\eta)-\tilde{z} \\
(\tau+\delta) m_{2} & =v_{1}(\tau+x+\delta+\eta)-\tilde{z} .
\end{aligned}
$$


Hence,

$$
m_{2}=v_{1}\left(\frac{\tau+x+\delta+\eta}{\tau+\delta}\right)-\frac{1}{\tau+\delta} \tilde{z}
$$

Substituting above

$$
0=\alpha-\beta \frac{1}{1-\gamma} \sum_{n=1}^{\infty} v_{n}+v_{1}\left(\frac{\gamma}{1-\gamma}-\alpha+\frac{\beta}{1-\gamma}\right)-v_{1}\left(\frac{\tau+x+\delta+\eta}{\tau+\delta}\right)+\frac{1}{\tau+\delta} \tilde{z}
$$

Note that

$$
\frac{\tau+x+\delta+\eta}{\tau+\delta}=\frac{\frac{\tau+x+\delta+\eta}{\tau+\delta+x}}{\frac{\tau+\delta}{\tau+\delta+x}}=\frac{1+\frac{\eta}{\tau+\delta+x}}{\frac{\tau+\delta}{\tau+\delta+x}}=\frac{1+\beta}{\alpha} .
$$

Hence, remembering again that $\alpha=1-\gamma$

$$
\begin{aligned}
0 & =\alpha-\beta \frac{1}{1-\gamma} \sum_{n=1}^{\infty} v_{n}+v_{1}\left(\frac{\gamma}{1-\gamma}-\alpha+\frac{\beta}{1-\gamma}\right)-v_{1} \frac{1+\beta}{\alpha}+\frac{1}{\tau+\delta} \tilde{z} \\
& =\alpha-\frac{\beta}{\alpha} \sum_{n=1}^{\infty} v_{n}+v_{1}\left(\frac{1-\alpha}{\alpha}-\alpha+\frac{\beta}{\alpha}\right)-v_{1} \frac{1+\beta}{\alpha}+\frac{1}{\tau+\delta} \tilde{z}
\end{aligned}
$$

Also

$$
\frac{1}{\tau+\delta}=\frac{\eta}{\tau+\delta} \frac{1}{\eta}=\frac{\frac{\eta}{\tau+\delta+x}}{\frac{\tau+\delta}{\tau+\delta+x}} \frac{1}{\eta}=\frac{\beta}{\alpha} \frac{1}{\eta}
$$

Hence,

$$
\begin{aligned}
0 & =\alpha-\frac{\beta}{\alpha} \sum_{n=1}^{\infty} v_{n}+v_{1}\left(\frac{1-\alpha}{\alpha}-\alpha+\frac{\beta}{\alpha}-\frac{1+\beta}{\alpha}\right)+\frac{\beta}{\alpha} \frac{1}{\eta} \tilde{z} \\
& =\alpha-\frac{\beta}{\alpha} \sum_{n=1}^{\infty} v_{n}+v_{1}\left(\frac{1-\alpha-\alpha^{2}}{\alpha}+\frac{\beta-1-\beta}{\alpha}\right)+\frac{\beta}{\alpha} \frac{1}{\eta} \tilde{z} \\
& =\alpha-\frac{\beta}{\alpha} \sum_{n=1}^{\infty} v_{n}+v_{1}\left(\frac{1-\alpha-\alpha^{2}}{\alpha}-\frac{1}{\alpha}\right)+\frac{\beta}{\alpha} \frac{1}{\eta} \tilde{z} \\
& =\alpha-\frac{\beta}{\alpha} \sum_{n=1}^{\infty} v_{n}+v_{1}\left(\frac{-\alpha-\alpha^{2}}{\alpha}\right)+\frac{\beta}{\alpha} \frac{1}{\eta} \tilde{z} \\
& =\alpha-\frac{\beta}{\alpha} \sum_{n=1}^{\infty} v_{n}-v_{1}(1+\alpha)+\frac{\beta}{\alpha} \frac{1}{\eta} \tilde{z}
\end{aligned}
$$

so that

$$
\begin{aligned}
\frac{\beta}{\alpha} \sum_{n=1}^{\infty} v_{n} & =\alpha-v_{1}(1+\alpha)+\frac{\beta}{\alpha} \frac{1}{\eta} \tilde{z} \\
\sum_{n=1}^{\infty} v_{n} & =\frac{\alpha^{2}}{\beta}-v_{1} \frac{\alpha(1+\alpha)}{\beta}+\frac{1}{\eta} \tilde{z}
\end{aligned}
$$

the other equation is 


$$
\sum_{n=1}^{\infty} v_{n}=\frac{\tilde{z}-v_{1}(\tau+\delta)}{\eta}=\frac{\tilde{z}}{\eta}-\frac{(\tau+\delta)}{\eta} v_{1}
$$

This implies that

$$
\begin{aligned}
\frac{\tilde{z}}{\eta}-\frac{(\tau+\delta)}{\eta} v_{1} & =\frac{\alpha^{2}}{\beta}-v_{1} \frac{\alpha(1+\alpha)}{\beta}+\frac{1}{\eta} \tilde{z} \\
-\frac{(\tau+\delta)}{\eta} v_{1}+v_{1} \frac{\alpha(1+\alpha)}{\beta} & =\frac{\alpha^{2}}{\beta} \\
v_{1}\left(\frac{\alpha(1+\alpha)}{\beta}-\frac{(\tau+\delta)}{\eta}\right) & =\frac{\alpha^{2}}{\beta} .
\end{aligned}
$$

Now recall that

$$
\frac{(\tau+\delta)}{\eta}=\frac{\frac{(\tau+\delta)}{\tau+\delta+x}}{\frac{\eta}{\tau+\delta+x}}=\frac{\alpha}{\beta} .
$$

This implies that

$$
\begin{aligned}
v_{1}\left(\frac{\alpha(1+\alpha)}{\beta}-\frac{(\tau+\delta)}{\eta}\right) & =v_{1}\left(\frac{\alpha(1+\alpha)}{\beta}-\frac{\alpha}{\beta}\right) \\
& =v_{1}\left(\frac{\alpha(1+\alpha)-\alpha}{\beta}\right)
\end{aligned}
$$

\section{E Computational Appendix}

\section{E.1 Product Size Distribution}

To solve the difference equation for the product distribution we truncate the product space from above an solve a system of linear equations.

From equation (25) we have

$$
\begin{gathered}
v(n+1)(n+1)(\tau+\delta)-v(n)(n(\tau+x+\delta)+\eta)+v(n-1)(n-1) x=0 \\
v(1)(\tau+x+\delta+\eta)-2(\tau+\delta) v(2)=z
\end{gathered}
$$

and the requirement that

$$
\sum_{n=1}^{\infty} v(n) n=1
$$

We solve this by truncating the system of equations at $n=\bar{n}$. We use $\bar{n}=20$ and $\operatorname{set} v(\bar{n})=0$. 
Write this in matrix form as

$$
\left[\begin{array}{cccccc}
1 & 2 & 3 & 4 & \cdots & \bar{n} \\
(\tau+x+\delta+\eta) & -2(\tau+\delta) & 0 & 0 & \cdots & 0 \\
0 & 2 x & -(3(\tau+x+\delta)+\eta) & 4(\tau+\delta) & \cdots & 0 \\
\vdots & \vdots & \vdots & \vdots & \ddots & \vdots \\
0 & 0 & 0 & 0 & \cdots & 1
\end{array}\right]\left[\begin{array}{c}
v(1) \\
v(2) \\
v(3) \\
\vdots \\
v(\bar{n})
\end{array}\right]=\left[\begin{array}{c}
1 \\
z \\
0 \\
\vdots \\
0
\end{array}\right]
$$

and then solve this.

Finally we have the entry rate from

$$
\text { Entry rate }=\frac{z}{\sum_{n=1}^{\bar{n}} v(n)}
$$

